\title{
BMAL1 coordinates energy metabolism and differentiation of pluripotent stem cells
}

\author{
Cristina Ameneiro ${ }^{1}$ (D), Tiago Moreira ${ }^{1}$ (D), Alejandro Fuentes-Iglesias ${ }^{1,2}(\mathbb{B}$, \\ Alba Coego $^{1} \mathbb{(}$, Vera Garcia-Outeiral $\mathbf{l}^{1,2} \mathbb{(}$, Adriana Escudero ${ }^{1,2} \mathbb{(}$, \\ Daniel Torrecilla ${ }^{1} \mathbb{0}$, Sonia Mulero-Navarro ${ }^{3}$ (D) Jose Maria Carvajal-Gonzalez ${ }^{3} \mathbb{D}$, \\ Diana Guallar ${ }^{1,4}$ (D) Miguel Fidalgo ${ }^{1,2}$ (])
}

\begin{abstract}
BMAL1 is essential for the regulation of circadian rhythms in differentiated cells and adult stem cells, but the molecular underpinnings of its function in pluripotent cells, which hold a great potential in regenerative medicine, remain to be addressed. Here, using transient and permanent loss-of-function approaches in mouse embryonic stem cells (ESCs), we reveal that although BMA L1 is dispensable for the maintenance of the pluripotent state, its depletion leads to deregulation of transcriptional programs linked to cell differentiation commitment. We further confirm that depletion of Bmal1 alters the differentiation potential of ESCs in vitro. Mechanistically, we demonstrate that BMAL1 participates in the regulation of energy metabolism maintaining a low mitochondrial function which is associated with pluripotency. Loss-offunction of Bmal1 leads to the deregulation of metabolic gene expression associated with a shift from glycolytic to oxidative metabolism. Our results highlight the important role that BMAL1 plays at the exit of pluripotency in vitro and provide evidence implicating a non-canonical circadian function of BMAL1 in the metabolic control for cell fate determination.
\end{abstract}

DOI 10.26508/lsa.201900534 | Received 23 August 2019 | Revised 31 March 2020 | Accepted 1 April 2020 | Published online 13 April 2020

\section{Introduction}

Circadian rhythms are necessary to coordinate key behavioural (e.g., sleep/wake cycle) and physiological (e.g., metabolism, hormone secretion, and stem cell homeostasis) processes in mammals (Bechtold \& Loudon, 2013; Lopez-Minguez et al, 2016; McAlpine \& Swirski, 2016; Weger et al, 2017; Dierickx et al, 2018). At the cellular level, the circadian clock is composed by transcriptional and translational feedback loops involving the clock master regulators BMAL1, CLOCK, PER, and CRY proteins, which ensure rhythmic gene expression to accommodate to the tissue and organ needs. Interestingly, although the proteins of the circadian clock are already present at early stages of embryonic development, circadian rhythms are not established until around the mid-gestation stage (Saxena et al, 2007; Umemura et al, 2017). In line with this, embryonic stem cells (ESCS), which are derived from the inner cell mass of the preimplantation blastocyst, are devoid of transcriptional circadian oscillations (Kowalska et al, 2010; Yagita et al, 2010; Umemura et al, 2014, 2017; Dierickx et al, 2017).

Given the lack of a compensating homologue in vivo, BMAL1 has been defined as the only essential component of the molecular circadian clock in mammals (Bunger et al, 2000). Bmal1 KO mice have impaired circadian behaviour and absence of rhythmicity in circadian target genes (Bunger et al, 2000). Moreover, they show infertility (Alvarez et al, 2008; Boden et al, 2010), show impaired glucose homeostasis (Rudic et al, 2004), and have been reported to have reduced life span and higher prevalence of age-related pathologies (Kondratov et al, 2006). Unexpectedly, many metabolic and age-related pathologies caused by Bmal1 depletion were not observed when using an inducible KO mouse model where Bmal1 depletion was performed in the adult age (Yang et al, 2016), suggesting important functions for this master regulator during embryogenesis. Given that BMAL1 is readily expressed in ESCS, even in the absence of a functional circadian clock, we hypothesized that additional roles of this factor in pluripotency remain to be discovered and could yield insights into its function during early stages of embryonic development.

To investigate the function of BMAL1 in pluripotent cells, which present a great therapeutic potential given their ability to generate cells of any adult tissue, we used transient and genetic models of Bmal1 loss-of-function in ESCS. We discovered that BMAL1 is dispensable for ESC maintenance, as its depletion does not affect pluripotency marker expression or colony formation. Nevertheless, we observed that ablation of Bmal1 in ESCs resulted in deregulation of genes from the three embryonic germ layers, and an aberrant

\footnotetext{
${ }^{1}$ Center for Research in Molecular Medicine and Chronic Diseases (CIMUS), Universidade de Santiago de Compostela (USC)-Health Research Institute (IDIS), Santiago de Compostela, Spain ${ }^{2}$ Department of Physiology, USC, Santiago de Compostela, Spain ${ }^{3}$ Department of Biochemistry, Molecular Biology and Genetics, Facultad de Ciencias, Universidad de Extremadura, Badajoz, Spain ${ }^{4}$ Department of Biochemistry and Molecular Biology, USC, Santiago de Compostela, Spain
} 
A
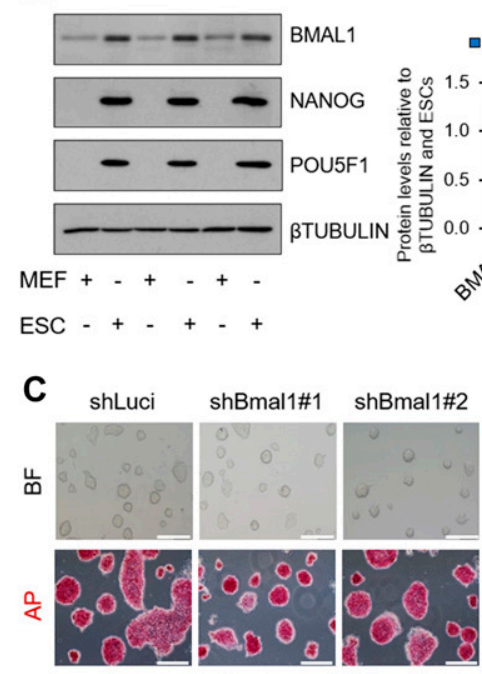

B

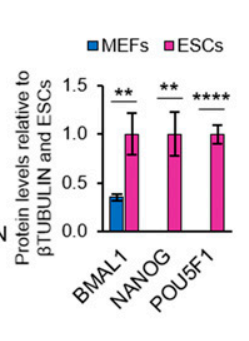

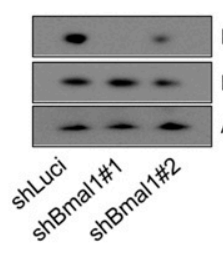

D

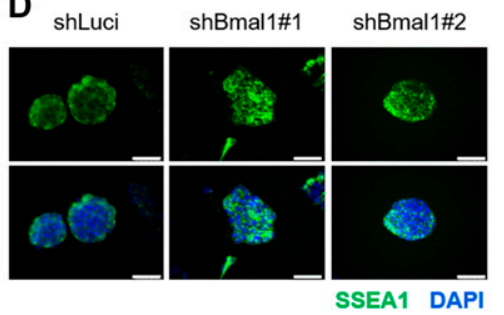

E

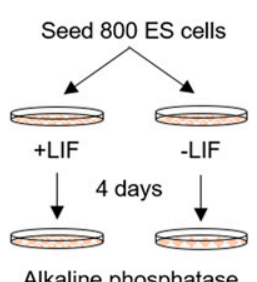

(AP) staining
$\mathbf{F}$

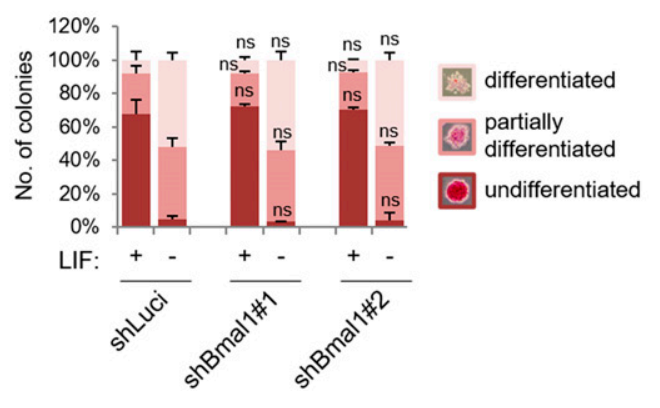

Figure 1. Silencing of Bmal1 does not compromise the maintenance of embryonic stem cells (ESCS).

(A) (Left) Western blot of BMAL1 and the pluripotency markers NANOG and POU5F1 in MEFs and ESCS. $\beta$ TUBULI $\mathrm{N}$ was used as loading control. Biological triplicates are shown. (Right) Quantification of the blots. (B) (Left) Western blot of BMAL1 and POU5F1 in ESCs transfected with shRNAs against Bmal1 or Luciferase as control. ACTIN (ACTB) was used as a loading control. One representative experiment is shown. (Right) Quantification of three independent experiments. (C) Bright-field (BF) and AP-staining images of ESCS transfected with shRNA against Bmal1 or Luciferase as a control. The scale bar represents $200 \mu \mathrm{m}$. (D) Immunofluorescence of the pluripotent marker SSEA1 in ESCs transfected with shRNAs against Bmal1 or Luciferase as control. Nuclei were stained with DAPI. The scale bar represents $50 \mu \mathrm{m}$. (E) Schematic representation for the colony formation assay. ESCS transfected with shRNA against Bmal1 or Luciferase as control are seeded at low confluence in standard medium with or without LIF, and colonies are counted and classified into the three indicated categories according to their AP-staining intensity. (F) Graphic representation for the counting and classification of colonies. $(n=3)$. In $(A, B, F)$, bars represent mean $\pm S D$. Two-tailed unpaired $t$ test analysis was performed. ${ }^{\star * * *} P<0.0001,{ }^{\star * \star} P<0.001,{ }^{\star *} P<0.01$; ns, not significant. Source data are available for this figure. induction of differentiation gene expression in vitro. Importantly, using embryonic organoids, we discovered that BMAL1 is necessary for in vitro gastruloid formation and proper expression of lineage specification markers. Mechanistically, we discovered that depletion of Bmall produced a change in metabolism-related genes and pathways, which are now considered to be drivers in the differentiation process. In particular, we observed a reduction in basal glycolysis and a concomitant increase in respiration, which was accompanied by an increase in mitochondrial reactive oxygen species (mtROS) production. Thus, our results uncover an unexpected function of BMAL1 in ESCS in metabolic regulation, where the clock is not yet "ticking," but BMAL1 function is already relevant for proper embryonic specification.

\section{Results}

Transient loss-of-function of BMAL1 is dispensable for ESC selfrenewal

To define the role of BMAL1 in pluripotent cells, which have been previously reported to lack circadian rhythms (Kowalska et al, 2010; Yagita et al, 2010; Umemura et al, 2014, 2017; Dierickx et al, 2017), we first determined the expression level of this core clock regulator in MEFs and pluripotent ESCS. Notably, when we analysed BMAL1 RNA and protein levels by RT quantitative PCR (RT-qPCR) and Western blot, respectively, we detected that its abundance was higher in ESCS compared with MEFs (Figs $1 \mathrm{~A}$ and S1A). In contrast, expression of Clock, another circadian regulator, was higher in MEFs, which have been reported to possess a functional circadian clock (Yagita et al, 2001) (Fig S1A). These data prompted us to consider whether BMAL1 may be important for pluripotency.

To understand the role of BMAL1 in ESCS, we first performed lossof-function experiments using two independent shRNAs against Bmal1 to reduce the likelihood of off-target effects. We confirmed the efficiency in silencing of Bmal1 compared with Luciferase control (shLuci) knockdown by analysing its protein levels by Western blot (Fig 1B). Importantly, we did not observe major differences in typical ESC morphology and AP staining in cells transduced with shRNAs against Bmal1 compared with shLuci (Fig 1C). Consistently, reduction of BMAL1 protein levels did not greatly impact pluripotency markers (i.e., Pouff1 and Nanog) at the mRNA level (Fig S1B) and/or the protein level (Fig 1B). Indeed, immunofluorescence assays revealed that silencing of Bmal1 does not alter expression variability and population heterogeneity of pluripotency markers (i.e., SSEA1, POU5F1, and ZFP281) in ESCS (Figs 1D and S1C). We next examined whether self-renewal properties of pluripotent cells were affected by the loss-of-function of Bmal1 in the presence 
A

\section{Bmal1 (Arnt)}

chr7:113280663-113313619

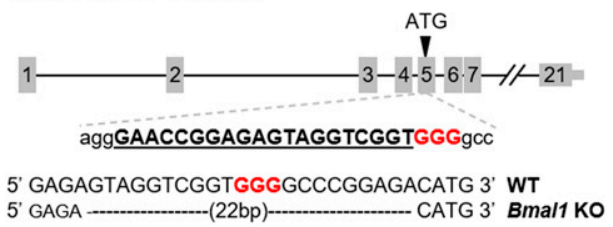

B
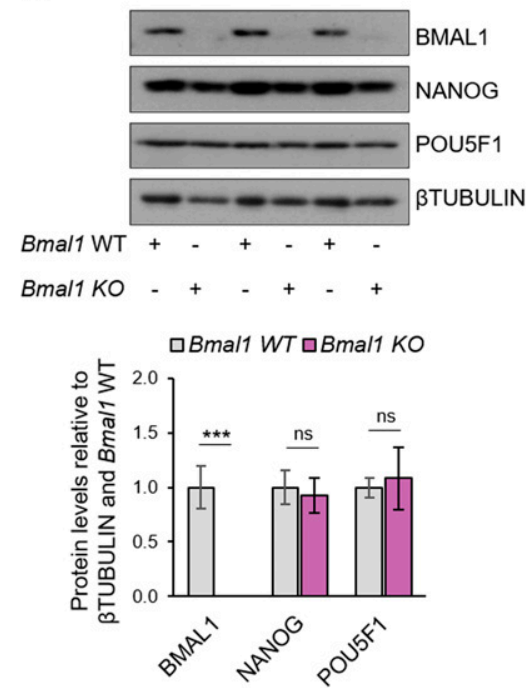

C

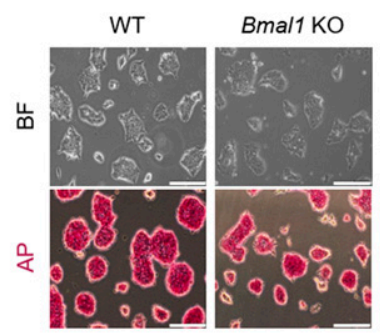

D

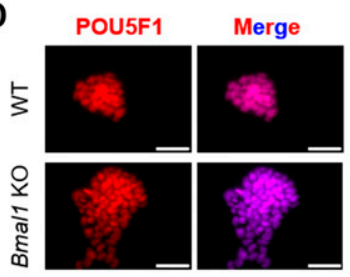

E

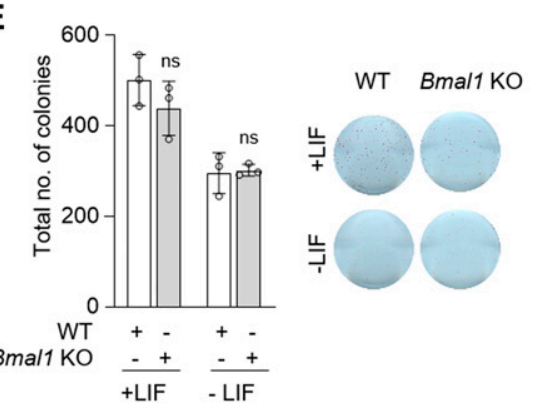

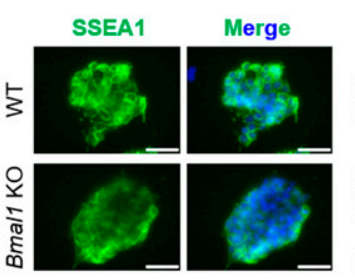

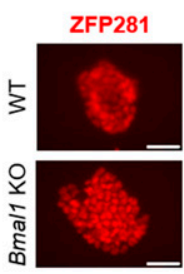

Merge

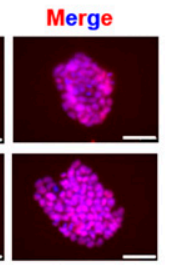

$\mathbf{F}$

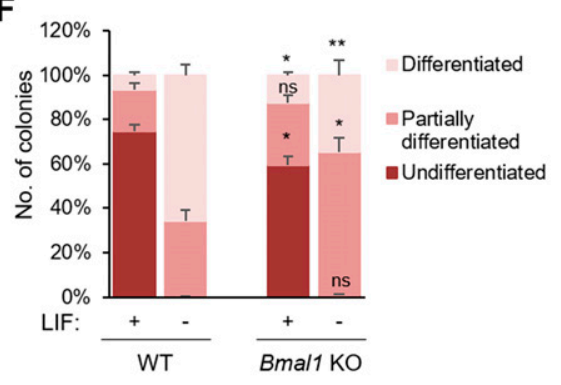

Figure 2. Generation and characterization of a CRI SPR/Cas9 Bmal1 KO embryonic stem cell (ESC) line. (A) (Top) Schematic representation for the CRISPR/C as9 strategy used. The designed sgRNA is underlined and the PAM sequence is highlighted in red. (Bottom)

Sequence deleted (22 bp) in Bmal1 KO alleles detected by Sanger sequencing. (B) (Top) Western blot of BMAL1 and the pluripotency factors NANOG and POU5F1 in Bmal1 WT and KO ESCS. $\beta$ TUBULIN was used as a loading control. (Bottom) Quantification of three independent experiments. (C) Bright-field (BF) and AP-staining images of Bmal1 WT and KO ESCs. The scale bar represents $200 \mu \mathrm{m}$. (D) Immunofluorescence of POU5F1, SSEA1, and ZFP281 in Bmal1 WT and KO ESCS. Nuclei were stained with DAPI. The scale bar represents $50 \mu \mathrm{m}$. (E) (Left) Number of ESC colonies formed in a clonogenic assay with Bmal1 WT and KO ESCs. (Right) Representative images of AP-stained wells are shown. (F) Graphic representation for the counting and classification of colonies of a colonyformation assay. $(n=3)$ In $(B, E, F)$, bars represent mean \pm SD. Two-tailed unpaired $t$ test analysis was performed. ${ }^{* \star *} P<0.001,{ }^{* \star} P<0.01,{ }^{*} P<0.05$; ns, not significant. Source data are available for this figure. or absence of leukaemia inhibitory factor (LIF). For this purpose, a reduced number of cells transduced with either the control shLuci or each of the two shRNAs against Bmal1 were plated and cultured at clonal density with or without LIF for $4 \mathrm{~d}$, followed by AP staining (Fig 1E). Consistent with the effect observed in bulk-grown ESCS, silencing of Bmal1 did not impact the proportion of undifferentiated, partially or fully differentiated colonies both in pluripotencysustaining (+LIF) or differentiation-promoting (-LIF) culture conditions (Fig 1F). Taken together, our results show that BMAL1 is dispensable for ESC self-renewal, despite being abundantly expressed in pluripotent cells.

\section{Absence of BMAL1 protein is compatible with pluripotency maintenance}

Given that knockdown with RNA interference can result in variable amounts of mRNA reduction, we aimed at generating ESC lines completely lacking Bmal1 expression to systematically dissect BMA $\mathrm{L} 1$ function in pluripotent cells. To this end, we used the CRISPR-C as9 nuclease system (Jinek et al, 2012; Cong et al, 2013) to generate a Bmal1 KO ESC line. First, we designed a sgRNA specifically targeting the start codon site of Bmal1 gene, located at the Exon 5 (Fig 2A). PCR genotyping and Sanger sequencing identified a Bmal1 KO ESC line (Figs $2 \mathrm{~A}$ and S2A-C). Western blot analysis confirmed complete depletion of BMAL1 protein in our selected ESC clone (Fig 2B).

To establish whether depletion of Bmal1 influences the maintenance of the pluripotent state of ESCS, we first examined the morphology and AP-staining pattern in Bmal1 KO ESCS. Notably, consistent with the Bmal1 knockdown data, Bmal1 KO ESCs displayed normal pluripotent colony morphology and stained positive for AP compared with WT pluripotent cells over the course of multiple passages (>10) (Fig 2C). In addition, the expression of pluripotency markers (i.e., Nanog, Pou5f1, and Zfp42) at the RNA and protein levels was not significantly affected in the absence of BMAL1 
A

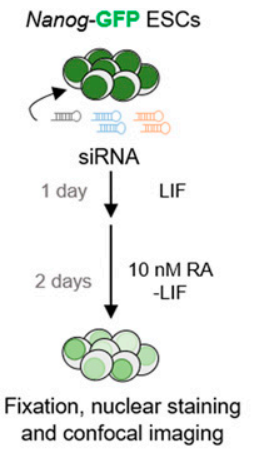

C

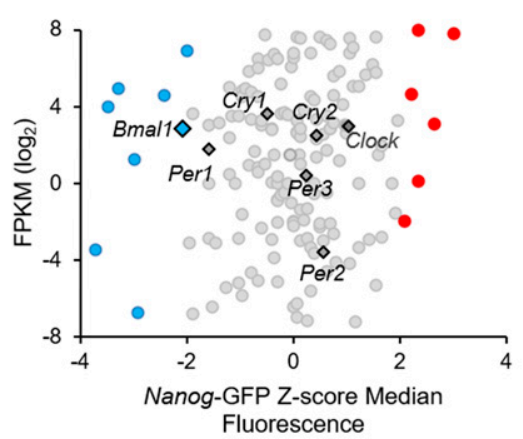

B

\begin{tabular}{|c|c|}
\hline Gene & Z-score \\
\hline Bmal1 & -2.0622 \\
Per1 & -1.5730 \\
Cry1 & -0.4111 \\
Per3 & 0.2616 \\
Cry2 & 0.4450 \\
Per2 & 0.5673 \\
Clock & 1.0565 \\
\hline
\end{tabular}

D

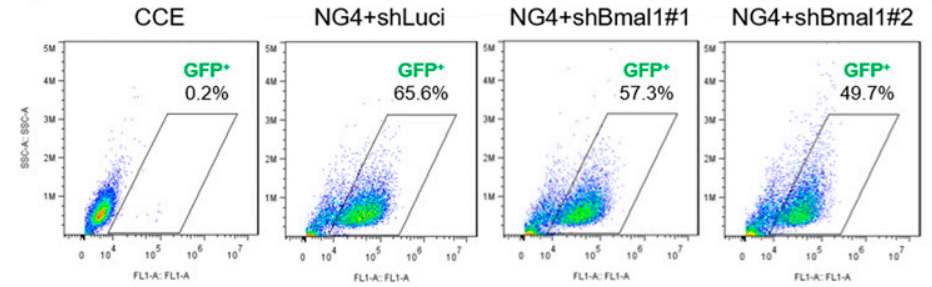

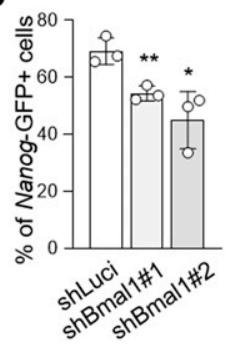

Figure 3. Absence of Bmal1 affects the exit from pluripotency.

(A) (Left) Schematic representation for screening conditions. Nanog-GFP (NG4) ESCs are transfected with siRNAs and cultured for $1 \mathrm{~d}$ in standard pluripotency medium followed by $2 \mathrm{~d}$ under mild retinoic acid (RA)-mediated differentiation. The cells are fixed, stained, and imaged. (Right) Scatter plot showing circadian clock core genes expression represented as FPKM and their effect in Nanog-GFP median fluorescence after screening conditions. (B) Summary table of Zscore median fluorescence values for circadian clock core genes after screening conditions highlighting Bmal1 as the gene with the highest effect among the core circadian regulators. (C) FACS analysis of Nanog-GFP intensity after RA-mediated differentiation of NG4 cells transfected with shRNA against Bmal1 or Luciferase as control. CCE parental ESC line is used as negative fluorescence control. The percentage represents the fraction of Nanog-GFP-positive cells in each sample in the indicated gate. One representative experiment is shown. (D) Percentage of Nanog-GFP-positive cells after transduction with the indicated shRNAs. $(n=3)$ Bars represent mean $\pm \mathrm{SD}$. Two-tailed unpaired $t$ test analysis was performed. ${ }^{* *} P<0.01,{ }^{*} P<0.05$.

Source data are available for this figure.
(Figs 2B and S2D and E). Moreover, immunofluorescence of key pluripotent markers (i.e., POU5F1, SSEA1, ZFP281, SOX2, and NANOG) (Figs 2D and S2F) suggested that pluripotency maintenance in Bmal1 KO ESCs was grossly unaffected. On the other hand, when we analysed the self-renewal capability of Bmal1-depleted ESCS, we did not observe changes in the number of colonies (Fig 2E) and only a mild reduction in the percentage of undifferentiated colonies $(74.5 \pm 3.3$ versus $59.6 \pm 3.9)$ in the presence of LIF (Fig 2F), which is in line with the acute silencing of Bmal1 using the shRNA approach (Fig 1F). Of note, Bmal1 KO ESCs showed a defect in differentiation upon LIF withdrawal as observed by the decrease in the percentage of fully differentiated colonies ( $65.6 \pm 4.7$ versus $34.7 \pm 6.7$ ) (Fig $2 F$ ), suggesting a possible role of BMAL1 during the exit of pluripotency. Collectively, taking together the knockdown and $\mathrm{KO}$ results, our data demonstrate that BMAL1 is dispensable for ESC maintenance.

\section{BMAL1 is required for exiting pluripotency in vitro}

Given that pluripotent cells can differentiate into all cell types from the three germ layers (i.e., ectoderm, endoderm, and mesoderm), we next interrogated the role of BMAL1 during the cellular differentiation process of ESCs. For this purpose, we first performed an in silico analysis using a genome-wide RNAi screen-published dataset for the identification of potential direct and indirect regulators of Nanog gene expression under mild retinoic acid (RA)-induced differentiation conditions, which could presumably be involved in the exit of pluripotency (Gingold et al, 2014). Remarkably, when we analysed the consequences of loss-of-function in 166 genes involved with circadian rhythms (GO:0007623), we noticed the existence of eight positive, including BMAL1, and six negative potential regulators of the expression of the pluripotency marker Nanog (Z score $>2$ ), measured by GFP fluorescent levels, which could play roles in the differentiation process (Fig 3A). Interestingly, we also observed the existence of opposing effects on Nanog promoter activity between the core circadian clock regulators BMAL1 and CLOCK (Fig 3B). Importantly, we further confirmed the regulatory effect of BMAL1 on Nanog promoter activity under the same RA-induced differentiation conditions, by flow cytometry analysis using the Nanog-GFP reporter ESC line NG4 (Schaniel et al, 2009) transduced with shRNAs against Bmal1 and shLuci as a control (Fig $3 \mathrm{C}$ and D). Future studies are warranted to investigate whether BMAL1 regulates Nanog promoter activity in a direct or indirect manner. Taken together, these results show that BMAL1 may be involved in early decisions during the exit of pluripotency and unveil the existence of specific functions of factors related with circadian processes in cell fate determination at the beginning of in vitro differentiation.

Next, to investigate the function of BMAL1 in differentiation, we analysed the ability of Bmal1 KO and wild-type (WT) ESCs to form teratomas. When subcutaneously injected in the flanks of immunodeficiency mice, both Bmal1 KO and WT ESCS were able to efficiently form teratomas of similar size (Fig 4A). Haematoxylin and eosin-stained sections of teratomas showed that a range of cell types and tissues from all three germ layers (i.e., mesoderm, ectoderm, and endoderm) was present in all teratomas regardless of BMAL1 presence (Fig 4B). These findings, together with our results showing that BMAL1 is dispensable for ESC maintenance, support that Bmal1 KO ESCS are pluripotent. It is possible that the role of BMAL1 in the exit of pluripotency observed in vitro (Fig 3) does not cause profound abnormalities which would be detectable during differentiation in vivo by teratoma assay. Thus, to determine if depletion of Bmal1 in ESCs can affect cellular differentiation in vitro, we performed embryoid bodies (EBs) assays, which mimic early embryonic development in a dish (Doetschman et al, 1985). After $6 \mathrm{~d}$ of culture in ESC medium without the LIF cytokine (Fig 4C), we observed that Bmal1 expression was down-regulated similarly to Nanog, concomitant with the up-regulation of markers from the three germ layers (i.e., Pax3, Gata6, and Mixl1) (Fig 4D). Consistent 
A
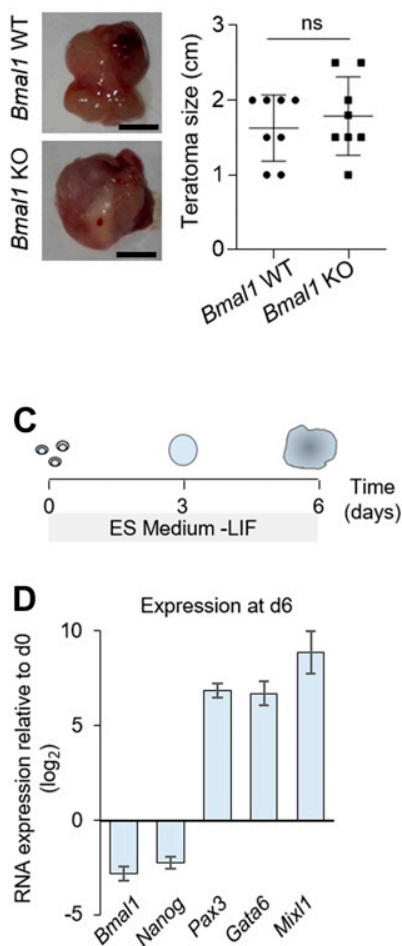

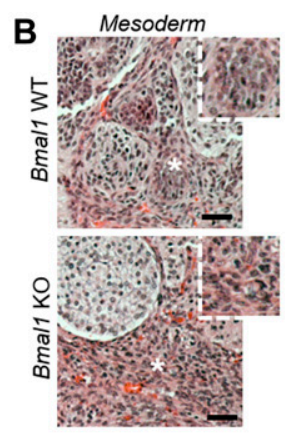

E
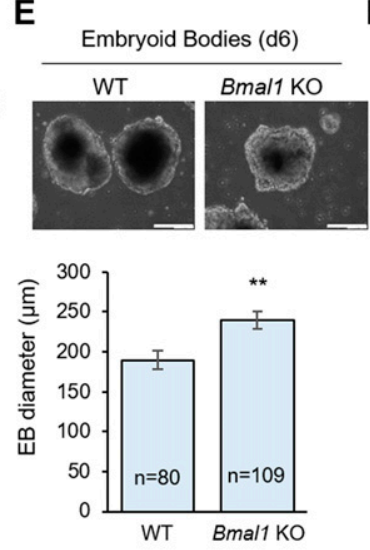

Ectoderm

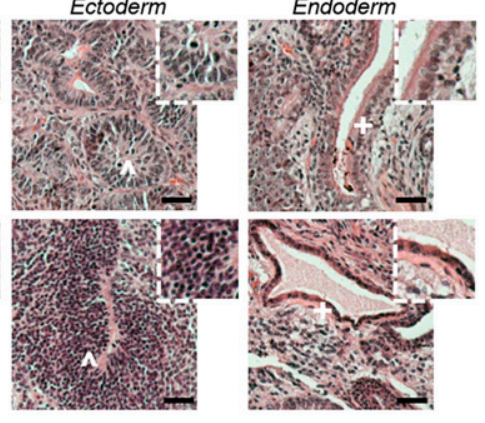

F

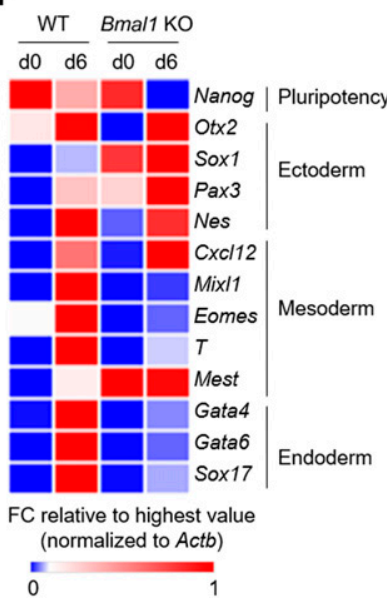

Figure 4. Bmal1 is required for embryonic stem cell in vitro differentiation.

(A) (Left) Teratomas formed by wild-type (WT) and Bmal1 KO ES cells after 3-4 wk of injection. The bar represents $5 \mathrm{~mm}$. (Right) Quantification of teratoma size ( $n=8$ tumours per genotype). (B) Histological analysis of teratomas of the indicated genotype by $\mathrm{H} \& \mathrm{E}$ staining. Structures representing the three embryonic germ layers in both genotypes were found. $\left({ }^{\star}\right)$, connective tissue; $\left({ }^{\wedge}\right)$, neural tissue; and $(+)$, epithelial tissue. $4 \times$ magnifications for each tissue are shown. The bar represents $30 \mu \mathrm{m}$. (C) Schematic representation for the embryoid body (EB) differentiation assay. (D) Relative expression of genes at day 6 of EB differentiation assay compared with day 0 in WT embryonic stem cells determined by RT-qPCR. Ectoderm-specific (i.e., Pax3), endodermspecific (i.e., Gata6), and mesoderm-specific (i.e., Mixl1) markers are shown. (E) (Top) Representative images of EBs at day 6 of differentiation in the indicated cell lines. The scale bar represents $200 \mu \mathrm{m}$. (Bottom) Quantification of the diameter of day 6 EBs for Bmal1 WT and $\mathrm{KO}$ cells. The number of EBs analysed is indicated. Data are shown as mean \pm SEM. (F) Relative expression of several germ layer-specific genes from day 0 and day 6 EBs determined by RT-qPCR. Expression fold changes are shown relative to the highest value. In $(A, D)$, data are shown as mean \pm SD Two-tailed unpaired $t$ test was performed. ${ }^{* *} P<0.01$; ns, not significant.

Source data are available for this figure. with their ability to generate teratomas, Bmal1 $\mathrm{KO}$ cells were able to form EBs (Fig 4E). However, we observed that Bmal1 KO EBs presented a significant increase in their size compared with the control (Fig 4E), suggesting intrinsic differences during the differentiation process through EB formation due to the absence of this master clock regulator. Moreover, when we analysed gene expression of several ectoderm, mesoderm, and endoderm markers, we found that they were differentially induced in Bmal1 $\mathrm{KO}$ at day 6, compared with WT EBs (Fig 4F). These results indicate that BMAL1 is required for ESC differentiation in vitro to properly establish germ layer-specific transcriptional programs.

\section{BMAL1 is important during in vitro gastrulation}

To further confirm our observation that loss of BMAL1 affects proper embryonic germ layer specification in vitro, we decided to use a recently reported gastruloid system (Beccari et al, 2018). Gastruloids are small aggregates of ESCs that undergo gastrulation-like events and elongation in vitro and mimic embryonic spatial and temporal gene expression (Beccari et al, 2018). Importantly, this gastrulation model can be used as an in vitro system to study early developmental events taking place in the mammalian embryo in vivo. Thus, we generated aggregates of WT or Bmal1 KO ESCS in N2B27 medium and subjected them to a pulse of a WNT agonist (i.e., CHIR99021) (Fig 5A). First, we analysed the expression pattern of WT gastruloids and observed that, similar to the pluripotency marker Nanog, Bmal1 was down-regulated at $120 \mathrm{~h}$ of gastruloid formation compared with ESCs $(t=0 \mathrm{~h}$ ), concomitant with germ layer marker induction (i.e., Pax3, Gata6, and Mixl1) (Fig 5B). We then compared the efficiency in the generation of gastruloids in the presence or absence of BMAL1. Remarkably, we observed that after $120 \mathrm{~h}$, the aggregates obtained from Bmal1 KO ESCS were significantly smaller (Fig 5C and D) and failed to elongate and polarize to give rise to gastruloid-like structures compared with wildtype cells (7.14\% versus $41.07 \%$, respectively) (Fig 5E). In addition, absence of BMAL1 during gastruloid formation was accompanied by altered expression of several lineage specification markers representative of three germ layers (Fig 5F). In particular, we observed deregulation, in the absence of BMAL1, of genes associated with in vivo gastrulation process such as Mixl1 and Eomes (Beccari et al, 2018). Likewise, we observed altered transcriptional dynamics of several members of the Hoxd gene cluster (Fig 5G), which is one of the hallmarks of axial gene regulatory systems whose sequential activation is associated with the patterning and formation during in vitro gastruloid formation (Beccari et al, 2018). Collectively, these results show that BMAL1 is required for efficient gastruloid formation in vitro and confirm that BMAL1 deficiency abrogates the correct induction of ectoderm, mesoderm, and endoderm markers during the exit of pluripotency.

\section{BMAL1 regulates transcriptional networks related to cellular differentiation}

To gain further insight into the molecular underpinnings of BMAL1 function in pluripotent cells, we performed transcriptional profiling 
A

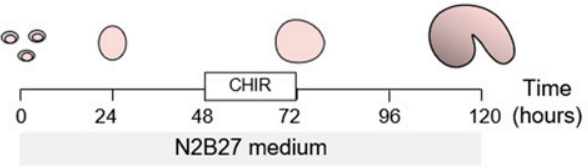

B

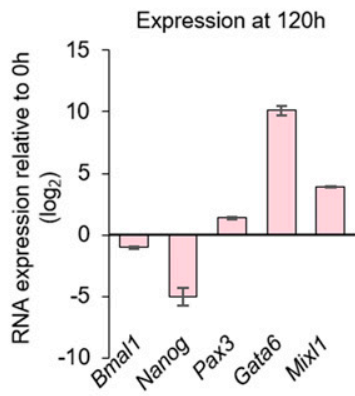

C

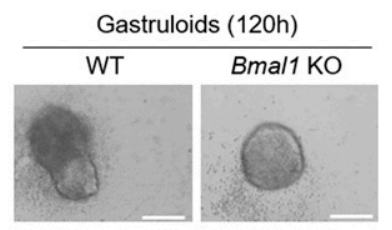

D
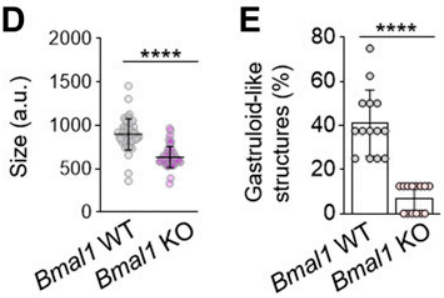

$\mathbf{F}$

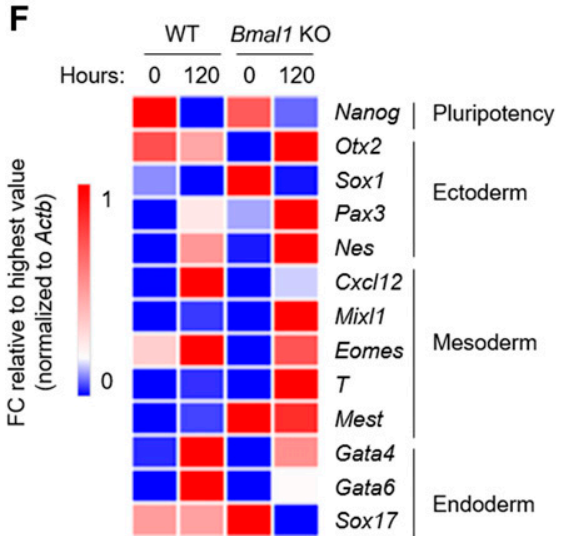

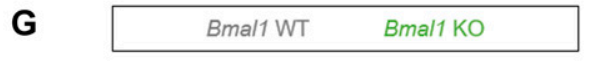

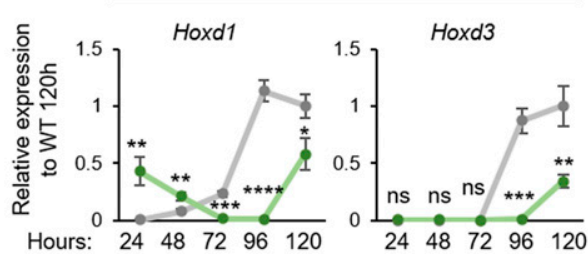

Hoxd4

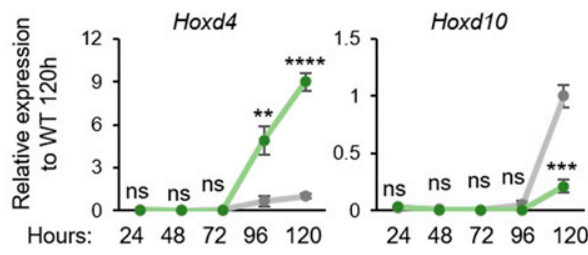

Figure 5. Ablation of Bmal1 disrupts gastrulation in vitro.

(A) Schematic depiction for the gastrulation assay performed (Beccari et al, 2018) for CHIR, GSK-3 inhibitor. (B) Relative expression of genes after $120 \mathrm{~h}$ of in vitro gastrulation assay of wild-type embryonic stem cells (ESCS) determined by RT-qPCR and expressed relative to ESCS (0 h). Ectoderm-specific (i.e., Pax3), endoderm-specific (i.e., Gata6), and mesodermspecific (i.e., Mixl1) markers are shown. (c) Representative images of $120 \mathrm{~h}$ aggregates for Bmal1 WT and KO cells. The scale bar represents $200 \mu \mathrm{m}$. (D) Quantification of the size of the aggregates formed by Bmal1 WT and KO cells at $120 \mathrm{~h}$ of in vitro gastrulation. (E) Percentage of gastruloid-like structures formed by Bmal1 WT and KO aggregates at $120 \mathrm{~h}$ of in vitro gastrulation $(\mathrm{n}=14$ independent experiments with at least eight formed aggregates per experiment). (F) Relative expression of Nanog and several germ layer-specific genes at the indicated time points of the gastruloid differentiation assay of WT and Bmal1 KO ESCs determined by RT-qPCR. Expression of fold changes are shown relative to the highest value. (G) Expression of Hoxd1, Hoxd3, Hoxd4, and Hoxd10 dynamics during in vitro gastrulation of Bmal1 WT and KO ESCS at the indicated time points. $(n=3)$. In $(B, D, E, G)$, data are represented as mean \pm SD. Two-tailed unpaired $t$ test was performed. ${ }^{\star * \star *} P<$ $0.0001,{ }^{* * *} P<0.001,{ }^{* *} P<0.01$; a.u., arbitrary units; ns, not significant.

Source data are available for this figure. of wild-type and Bmal1 KO ESCs. We identified 444 up-regulated and 197 down-regulated genes that were significantly changed with a difference in abundance greater than twofold in the absence of BMAL1 (Fig 6A and Table S1). Remarkably, Gene Ontology (GO) analysis of these misregulated genes upon Bmal1 depletion showed enrichment for differentiation processes (Fig 6B). To validate these results, we performed RT-qPCR analysis of a number of up-regulated (e.g., Tead4 and Mest) and down-regulated (e.g., Eomes and Snai3) early cell fate markers upon Bmal1 KO in ESCS (Fig $6 C$ and D). Moreover, gene set enrichment analysis (GSEA) also identified the up-regulation of gene signatures related to stem cell differentiation in the absence of BMAL1 function when compared with their wild-type counterparts (Fig 6E). Thus, these results suggest that Bmal1 depletion leads to transcriptional changes in genes related to developmental processes by direct or indirect mechanisms, which is in line with our results showing the requirement of this circadian master regulator for proper cellular differentiation of ESCs. Indeed, we observed that depletion of Bmal1 led to a significant deregulation of genes related to the three embryo germ layers (i.e., endoderm, ectoderm, and mesoderm) in ESCS, whereas housekeeping genes remained unaltered (Fig 6F). Importantly, in spite of these global transcriptional changes, the GSEA analysis further confirmed the pluripotent cell identity of Bmal1 KO ESCs denoted by no significant changes in the expression of ESC-enriched genes as well as targets of the core pluripotency regulators POU5F1 (OCT4), SOX2, and NANOG (OSN) (Fig S3A). Taken together, our data suggest that BMAL1 loss may influence the differentiation potential of ESCs in vitro by altering the expression of early specification genes of the three germ layers in pluripotent cells.

\section{BMAL1 supports glycolytic metabolism in ESCs}

To understand how BMAL1 orchestrates transcriptional programs involved in proper cell differentiation in vitro, we next compared the gene expression changes after Bmal1 depletion in ESCS with those caused by loss-of-function of other transcription regulators using the Network2Canvas computational tool (Tan et al, 2013). Interestingly, we found a close correlation of the transcriptional changes in the absence of BMAL1 with those upon depletion of the master pluripotency regulator POU5F1 (Kim et al, 2015) as well as other pluripotent regulators related with differentiation and/or cellular metabolism, including ZFX (Galan-Caridad et al, 2007; Chen et al, 2008) and Polycomb members (i.e., EED and SUZ12) (Brookes et al, 2012; Di Croce \& Helin, 2013) (Fig S3B). These results, together with the observation that the expression of these factors was not altered upon Bmal1 depletion in mouse ESCs (Figs 2B and $\mathrm{S} 3 \mathrm{C}$ and $\mathrm{D}$ ), suggest the existence of common pathways regulated 
A

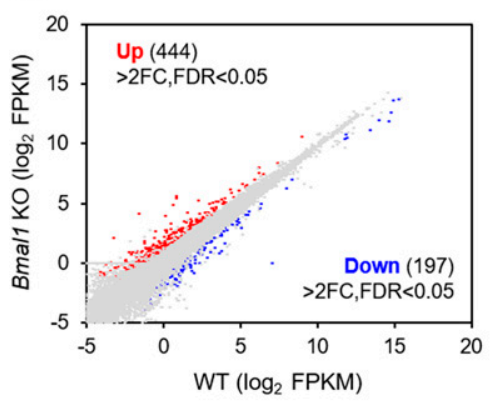

B

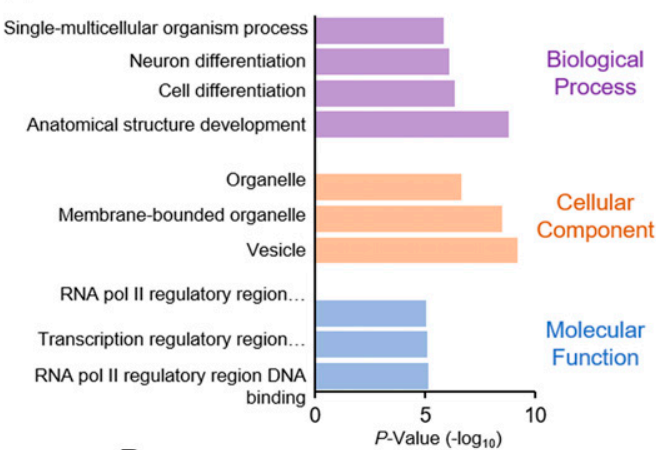

C
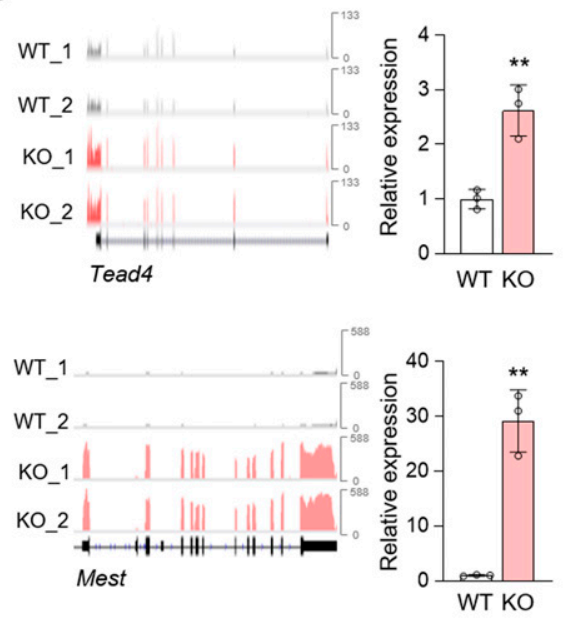

E

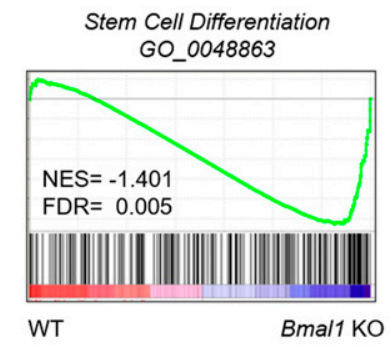

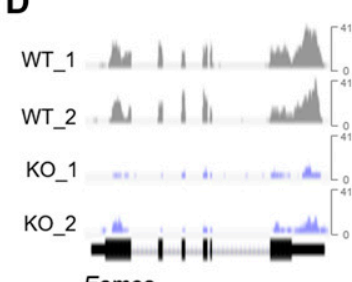

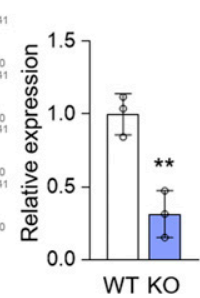

$\underline{\mathrm{GO}}$ analysis of misregulated genes

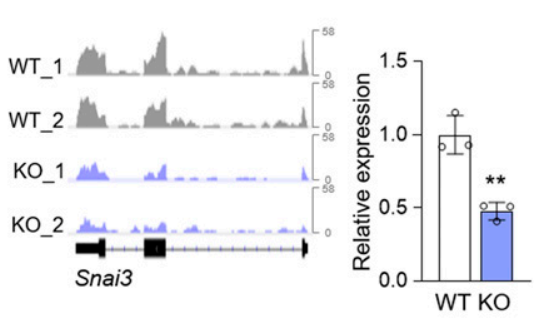

$\mathbf{F}$

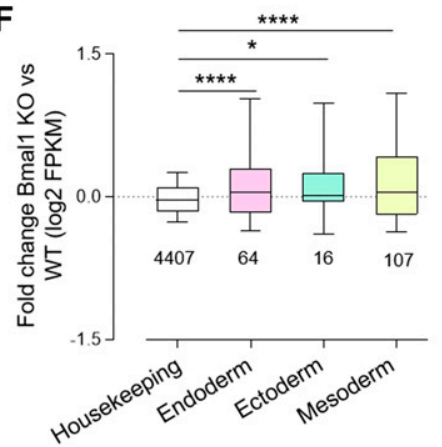

Figure 6. Bmal1 participates in the regulation of cell differentiation transcriptional programs in embryonic stem cells (ESCs).

(A) Scatter plot of gene expression (in $\log _{2}$ FPKM) in Bmal1 KO versus wild-type (WT) control ESCS determined by RNA-seq. Biological duplicates were analysed. Genes significantly (false discovery rate [FDR] $<0.05$ ) up-regulated and down-regulated more than twofold are shown in red and blue, respectively. (B) Gene Ontology (GO) analysis of genes misregulated in Bmal1 KO ESCs. The $x$-axis corresponds to the negative $\log _{10} P$-values. (C) (Left panel) Integrative genome viewer representation of RNA-seq reads for representative up-regulated genes in Bmal1 KO cells. (Right panel) RT-qPCR analysis validating the RNA-seq results $(n=3)$. (D) (Left panel) Integrative genome viewer representation of RNA-seq reads for representative down-regulated genes in Bmal1 KO cells. (Right panel) RT-qPCR analysis validating the RNAseq results $(n=3)$. $(E)$ Gene set enrichment analysis from WT and Bmal1 KO RNA-seq data against a stem cell differentiation gene set. Normalized enrichment score (NES) and FDR are shown. (F) Fold RNA expression changes of the indicated gene categories in WT and Bmal1 KO ESCs. The number of genes included in each category is indicated. Whiskers extend to the $10^{\text {th }}-90^{\text {th }}$ percentile range. In (C, D), data are shown as mean \pm SD. Two-tailed unpaired $t$ test was performed. ${ }^{* \star * *} P<0.0001,{ }^{* *} P<0.01,{ }^{*} P<0.05$.

Source data are available for this figure. by this set of factors and BMAL1. Indeed, Kyoto Encyclopedia of Genes and Genomes (KEGG) pathway analysis of the misregulated genes in Bmal1 KO ESCS revealed an enrichment in metabolicassociated processes (Fig S4A). Notably, among the most differentially expressed genes (false discovery rate [FDR] $>0.05$ and fold change $>2$ ), we found 19 down-regulated and 49 up-regulated metabolism-related genes in Bmal1-depleted cells (Fig S4B). In particular, we observed that BMAL1 loss influences the expression of several genes related with mitochondrial complex I-V and the tricarboxylic acid cycle or Krebs cycle (Figs 7A and S4C). Collectively, our results show that BMAL1 contributes to the proper transcriptional landscape regulation of pluripotent cells and that its depletion leads to the deregulation of metabolism-related transcriptional pathways. Changes in metabolic activity are closely linked to the exit of pluripotency, partly by influencing the epigenome during cell commitment (Cliff \& Dalton, 2017; Dahan et al, 2019). Thus, we speculated that Bmal1 depletion can alter early cell differentiation potential through changes in the expression of metabolic gene networks that govern the balance between glycolytic and oxidative phosphorylation (OXPHOS) activity. In agreement with our hypothesis, we found that Bmal1 KO cells showed reduced basal glycolysis compared with WT cells (Fig 7B). Conversely, depletion of Bmal1 in ESC $\mathrm{s}$ led to an increase in the basal oxygen consumption rate (OCR) (Fig 7C). Thus, we observed a metabolic switch in ESCs caused by Bmal1 depletion, which was translated into a more oxidative versus glycolytic use of glucose in Bmal7 KO ESCS compared with WT ones (Fig 7D). Although there is usually a tight coupling between electron transport and ATP synthesis, under certain conditions, protons can 
A
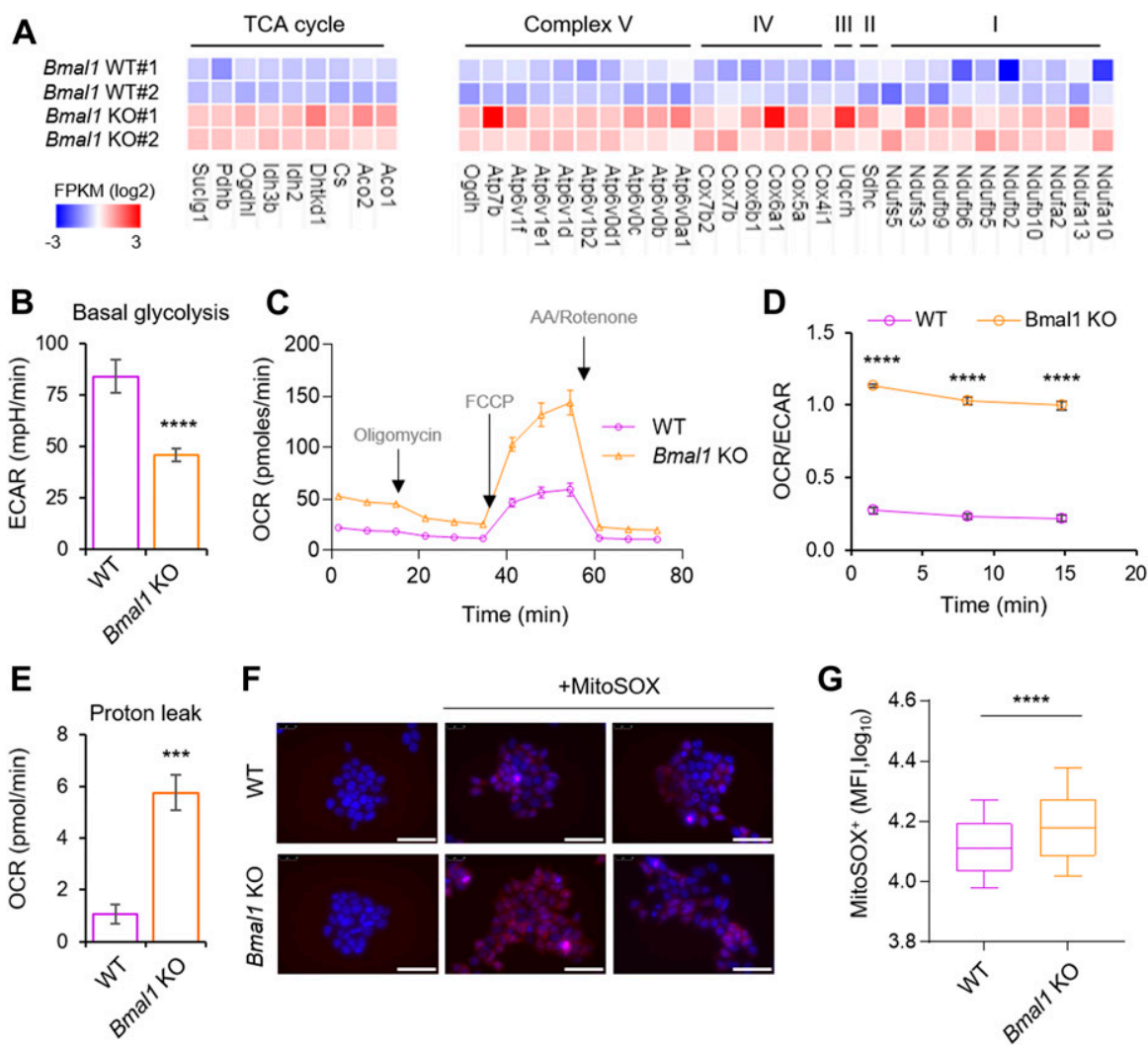

F
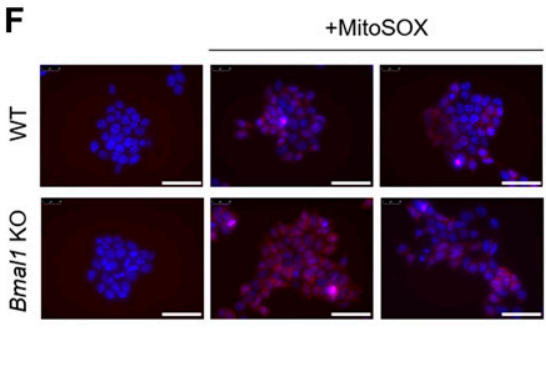

G
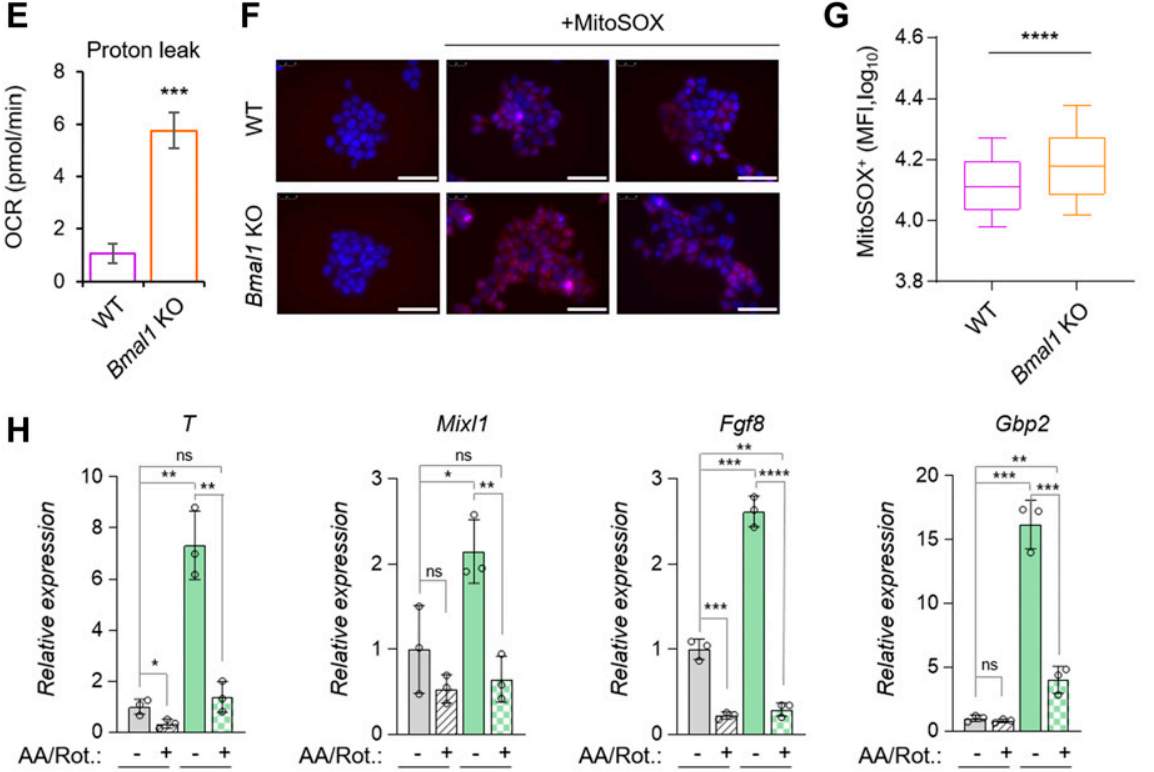

AA/Rot:: $\stackrel{-+\ldots+}{+}$

Bmal1 WT KO

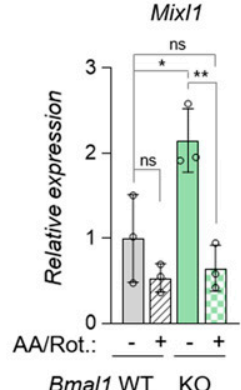

Bmal1 WT KO

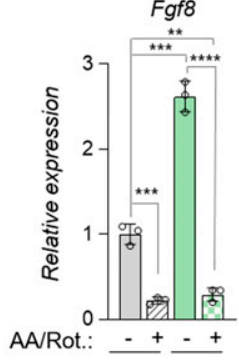

Bmal1 WT KO

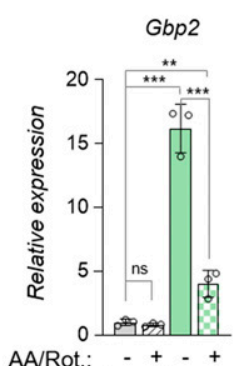

AA/Rot:: $\frac{-++}{\mathrm{K} O}$
Figure 7. Depletion of Bmal1 alters metabolic gene pathways, causing an increase in respiration and mitochondrial ROS production in embryonic stem cells (ESCs).

(A) Heat map of the expression of tricarboxylic acid cycle (TCA) and mitochondrial complex I-V genes from wild-type (Bmal1 WT) and Bmal1 KO ESCs RNA-seq. (B) Basal glycolysis levels determined as extracellular acidification rate (ECAR) in wild-type (WT) and Bmal1 $\mathrm{KO} \operatorname{ESCS}(n=3)$. (C) Bioenergetics assays in ESCs from WT and Bmal1 KO ESCS. The different drugs used in the assay are indicated in grey $(n=3)$. (D) OCR/ECAR under basal conditions for the indicated cell lines at three different time points $(n=3)$. (E) Proton leak shown as OCR in WT and Bmal1 KO ESCS $(n=3)$. (F) Fluorescence representative microscopy images showing MitoSOX fluorescence (red) in WT and Bmal1 KO ESCS. Nuclei were stained with DAPI and shown in blue. The scale bar represents $50 \mu \mathrm{m}$. (G) Box plots showing the mean fluorescence intensity of MitoSOX treated Bmal1 WT and KO cells. Center lines, medians; whiskers extend to the $10^{\text {th }} / 90^{\text {th }}$ percentile. $(\mathbf{H})$ Relative expression of the indicated genes in Bmal1 WT and KO cells with or without antimycin A/rotenone (AA/Rot) treatment. $t$ test was performed. $(n=3)$. In $(B, C, D, E, H)$, data are represented as mean $\pm \mathrm{SD}$. Two-tailed unpaired $t$ test was performed. ${ }^{* * * *} P<0.0001,{ }^{* * *} P<0.001$, ${ }^{* *} P<0.01,{ }^{*} P<0.05$; AA, Antimycin A; FCCP, carbonyl cyanide4 phenylhydrazone; ns, not significant; OCR, oxygen consumption rate.

Source data are available for this figure. re-enter the mitochondrial matrix with no contribution to ATP generation, in a process known as mitochondrial uncoupling or proton leak (Cadenas, 2018). Indeed, we observed that the increase of basal oxidative metabolism in cells lacking BMAL1 (Fig S4D) was accompanied by an increase in proton leak (Fig 7E) and a reduction in the coupling efficiency of mitochondria (Fig S4E). Although the exact link between proton leak and mtROS production is controversial, it is now clear that there is a mutual connection between these two processes (Nanayakkara et al, 2019). To interrogate the effect of Bmal1 depletion on the production of mtROS species, we stained WT and Bmal1 KO ESCs with MitoSOX Red. Interestingly, we observed that ESCs lacking BMAL1 displayed a significant increase in mtROS levels compared with WT cells (Figs 7F and G and S4F). Collectively, these findings show that BMAL1 is required for proper metabolic dynamics and mitochondrial function of pluripotent ESCS. Taking into account that the balance between glycolysis and OXPHOS is critical for modulating the differentiation potential of pluripotent cells (Wu et al, 2016; Cliff \& Dalton, 2017; Zhang et al, 2018; Dahan et al, 2019), we tested whether reducing OXPHOS activity in Bmal1 KO ESCs could restore the proper expression of lineage specification markers during in vitro differentiation. For this purpose, we used an early differentiation in vitro assay to rapidly induce the expression of genes involved in mesendoderm (ME) lineage choice (Thomson et al, 2011). In line with our previous differentiation assays, absence of BMAL1 significantly alter the expression of lineage markers upon ME differentiation (Fig $7 \mathrm{H}$ ). Importantly, presence of mitochondrial respiration inhibitors (i.e., antimycin A/rotenone [AA/Rotenone]) during ME differentiation of Bmal1 KO ESCS was sufficient to significantly rescue the expression of T, Mixl1, Fgf8, and Gpb2 lineage markers compared with Bmal1 WT ESCS (Fig 7H). These data further suggest that BMAL1 influences early cell fate specification during in vitro differentiation through the modulation of OXPHOS activity in ESCS. 


\section{Discussion}

Here, we demonstrate that BMAL1 is not required for ESC maintenance. In contrast, we show that this core component of the circadian clock plays critical roles during ESC in vitro differentiation. Indeed, depletion of Bmal1 caused the deregulation of genes from the three germ layers (i.e., ectoderm, mesoderm, and endoderm). In line with this, we show that the absence of BMAL1 affects EB formation and gastrulation, using two independent differentiation in vitro organoid systems. Similar conclusions were drawn from an independently performed study, which recently came to our attention (Gallardo et al, 2020). Mechanistically, we find that depletion of Bmal1 induces a shift in the basal metabolism of pluripotent cells involving an increase OXPHOS activity accompanied by augmented mtROS species production. Importantly, decreasing OXPHOS activity is sufficient to restore proper induction of lineage-specific marker expression during mesendoderm differentiation in the absence of BMAL1.

For circadian oscillation to occur, CLOCK and BMAL1 need to heterodimerize to activate their downstream transcriptional targets, which include Per1/2/3 and Cry $1 / 2$ (Takahashi, 2017). In agreement with the observation that circadian transcriptional oscillations are not functional in ESCS (Kowalska et al, 2010; Yagita et al, 2010; Umemura et al, 2014, 2017; Dierickx et al, 2017), when we analysed Cry1/2 and Per1/ 2/3 expression in Bmal1 $\mathrm{KO}$ cells, we did not observe any significant change in their transcriptional levels (Table S1), further supporting a clock-independent function for BMAL1 in pluripotent stem cells. Several reports have demonstrated that circadian oscillations are absent from pluripotent cells by using bioluminescent reporter systems and analysing of clock gene expression (Kowalska et al, 2010; Yagita et al, 2010; Umemura et al, 2014, 2017; Dierickx et al, 2017). Nevertheless, both BMAL1 and CLOCK proteins have been reported to be present in ESCS by us (this study) and others (Lu et al, 2016), respectively, posing the question of how circadian oscillations are inhibited in pluripotent stem cells. One possible explanation was indicated by a study that demonstrated that in ESCS, PER is retained in the cytoplasm, therefore avoiding the proper nuclear function of the negative feedback loop required for cyclic circadian regulation (Umemura et al, 2014). Moreover, in contrast to Lu and colleagues (Lu et al, 2016), a recent study claimed that despite being expressed at the mRNA level, CLOCK protein was absent in ESCs because of microRNAmediated posttranscriptional repression (Umemura et al, 2017). Although the absence of transcriptional cycling in pluripotent cells seems faithfully demonstrated, the extent to which each of the proposed mechanisms contributes to inhibit the normal function of the clock remains to be clarified. Importantly, CLOCK and BMAL1 seem to have opposite non-overlapping functions in terms of ESC differentiation (Gingold et al, 2014). This is in agreement with our observation that Bmal1 depletion facilitated the down-regulation of the pluripotency factor Nanog in conditions of mild differentiation, whereas Clock appeared to have the opposite effect, in agreement with what Lu and colleagues showed previously (Lu et al, 2016). Future studies are required to scrutinize common and specific roles of CLOCK and BMAL1 in ESCS, and additional levels of regulation that can shed light into the mechanisms by which pluripotent cells lack functional circadian rhythms.
Previous reports on the effect of loss of Bmal1 expression on early embryo development are not consistent. Whereas Bradfield et al reported a normal mendelian ratio of $\mathrm{Bmal1}^{-/-}$pups being born from the mating of two heterozygous $\mathrm{Bmal1}^{+/-}$mice (Bunger et al, 2000), several recent reports have shown that Bmal1 KO zygotes show reduced blastocyst formation and post-implantation development (Xu et al, 2016, 2017). Importantly, these defects could be directly associated with intrinsic developmental issues, given that they used females with at least one Bmal1 allele, which have been reported to possess normal reproduction physiology (Xu et al, 2017). Our observation that depletion of Bmal1 in ESCS, which are equivalent to the inner cell mass of the preimplantation blastocyst (around E3.5), show defects during in vitro differentiation to the three germ layers (i.e., ectoderm, endoderm, and mesoderm) would further support an embryo-autonomous role for BMAL1 in embryonic development. Taking into consideration the sterility issues described both in male and female Bmal1 KO mice (Alvarez et al, 2008; Boden et al, 2010; Ratajczak et al, 2012; Liu et al, 2014; Xu et al, 2016), the in vivo study of Bmal1 KO embryos is limited by the need of crossing heterozygous progenitors. We propose that ESC differentiation through gastruloid aggregation represents a powerful platform to investigate BMAL1 function in early stages of development where circadian rhythms have not yet been established. Moreover, this system allows to differentiate embryo-autonomous circadian mechanisms, excluding the influence from central and other periphery circadian clocks, which are also affected by the disruption of BMAL1 function.

In this study, we demonstrate for the first time a non-canonical function of BMAL1 in cells lacking circadian oscillations. This is in line with the observation that conditional depletion of Bmal1 at the adult age does not recapitulate many of the metabolic and agerelated pathologies observed in Bmal1 KO mice (Yang et al, 2016). This observation implies that BMAL1 may have several important circadian-dependent and circadian-independent functions during embryogenesis. Importantly, the higher age-dependent accumulation of reactive oxygen species (ROS) observed in several tissues of Bmal1 KO animals (Kondratov et al, 2006) could explain the early onset of age-associated pathologies observed in these animals. In line with this, we observed an increase in the mtROS levels in ESCS lacking Bmal1, even when the clock is not yet active, pointing to a possible factor contributing to the aging phenotypes observed in these animals later during their lifetime. Moreover, previous studies have demonstrated that oxidative stress negatively impacts oocyte quality and fertilization, together with embryo development (Matsuzuka et al, 2005; Tamura et al, 2008). Thus, the decrease in these parameters observed in $\mathrm{Bmal1}^{-/-}$in vivo may not only be caused by the reproductive female organs but also from excess of ROS at the embryo level.

Finally, we observed that ablation of Bmal1 in ESCs affected their metabolism by reducing glycolysis and increasing oxidative phosphorylation (OXPHOS). In line with this observation, using genome-wide immunoprecipitation-based techniques, BMAL1 has been shown to target genes related to cellular metabolism in somatic cells (Hatanaka et al, 2010; Wu et al, 2017; Reinke \& Asher, 2019), therefore pointing to a potential direct regulation of metabolic genes by BMAL1 through chromatin binding. Although until recently changes in metabolism were thought to be a consequence 
of differentiation, it is now clear that metabolism plays an active role in cell fate commitment, mainly by modifying the epigenome, which can in turn regulate pluripotency, differentiation, and somatic cell reprogramming (Dahan et al, 2019). Pluripotent stem cells favour glycolysis over OXPHOS, thus both reducing potential DNA damage by ROS, whereas increasing the amount of available intermediate metabolites for biosynthesis of lipids and nucleotides. Differentiation of ESCS shows a switch towards a more oxidative metabolic status, and interfering with glycolysis or OXPHOS processes has been shown to interfere with lineage commitment (Teslaa \& Teitell, 2015; Cliff \& Dalton, 2017). In line with this, we observed that BMAL1 is not only required for proper metabolic gene expression but also for appropriate induction of differentiation by EB or gastruloid formation. In agreement with this observation, we observed that global gene expression profile induced by Bmal1 depletion was significantly similar to those of loss-offunction of pluripotent factors involved in metabolic regulation such as POU5F1, ZFX, or PRC2 members (i.e., EED and SUZ12) (GalanCaridad et al, 2007; Chen et al, 2008; Brookes et al, 2012; Di Croce \& Helin, 2013; Kim et al, 2015). Future studies will be needed to dissect to what extent BMAL1 metabolic targets at the chromatin level are overlapping or different between pluripotent and somatic cells. Intriguingly, ESCS have been shown to present an oscillatory behaviour in glucose transporter expression (Paulose et al, 2012) even before the onset of a functional circadian clock. These data, together with our study, open up a new exciting field to interrogate circadianindependent BMAL1-mediated cyclic regulation of metabolism.

In sum, this novel function of BMAL1 in pluripotency exiting through metabolic regulation opens new avenues for exploring the involvement of non-canonical circadian clock regulation in development and disease.

\section{Materials and Methods}

\section{Cell lines and cell culture}

ESCS were cultured on $0.1 \%$ gelatin-coated plates at $37^{\circ} \mathrm{C}$ with $5 \%$ $\mathrm{CO}_{2}$ and maintained in DMEM (high glucose, D6429; Sigma-Aldrich), containing 15\% FBS (10270-106; Gibco), 2 mM L-glutamine (G7513; Sigma-Aldrich), $1 \times$ nonessential amino acids (11140; Gibco), 1\% nucleoside mix, 1× penicillin/streptomycin (15140; Gibco), $10^{-4} \mathrm{M}$ 2-mercaptoethanol (M6250; Sigma-Aldrich), and 1,000 $\mathrm{U} / \mathrm{ml}$ of recombinant LIF.

HEK293T cells were cultured at $37^{\circ} \mathrm{C}$ with $5 \% \mathrm{CO}_{2}$ in DMEM (high glucose, D6429; Sigma-Aldrich), containing 10\% FBS (10270-106; Gibco), 2 mM L-glutamine (SH30034.01; GE Healthcare), 1× penicillin/ streptomycin (15140; Gibco), and $10^{-4}$ M 2-mercaptoethanol (M6250; Sigma-Aldrich).

\section{RNA extraction}

Total RNA was extracted using the E.Z.N.A. Total RNA Kit I kit (R683402; Omega Bio-Tek) following the manufacturer's instructions.

For RNA sequencing (RNA-seq), the RNA samples were extracted using Trizol (15596018; Invitrogen) and Phasemaker Tubes (A33248; Invitrogen) following the manufacturer's instructions.

\section{RT-qPCR analysis}

RNA was converted into cDNA using qSCRIPT (84034; Quanta). Quantitative PCR was performed using the PowerUp SYBR Green Master Mix (4367659; Thermo Fisher Scientific) on the StepOnePlus Real-Time PCR System (Applied Biosystem). Gene-specific primers used are provided in Table S2.

\section{ShRNA design, lentivirus production, and cell transduction}

ShRNA sequences specific for mouse transcripts were found in the GPP Web Portal Tool available at https://portals.broadinstitute.org/ gpp/public/. Oligos targeting the Luciferase and Bmal1 transcripts were cloned in a lentiviral pLKO.1 puro plasmid. ShRNA-targeted sequences used in this study are shown in Table S2. pLKO.1 puro was a gift from Bob Weinberg (plasmid \#8453; Addgene; http://n2t.net/ addgene:8453; RRID: Addgene 8453) (Stewart et al, 2003).

For lentiviral production, eight million HEK293T cells were seeded in 150-mm plates and transfected $24 \mathrm{~h}$ later with $20 \mu \mathrm{g}$ of the corresponding pLKO.shRNA plasmid together with $10 \mu \mathrm{g}$ of psPAX2 and pMD2.G packaging mix using PEI (Polyethylenimine, 408727; Sigma-Aldrich) following the manufacturer's instructions. The next day, fresh medium was added to the cells, and viral supernatants were collected 48 and $72 \mathrm{~h}$ after transfection. Viruses were then concentrated using centrifugal filter units with $0.22-\mu \mathrm{m}$ pore size (UFC903024; Amicon).

\section{AP staining}

The presence of AP enzyme is characteristic of undifferentiated cells (Stefkova et al, 2015) and is, therefore, routinely used as a pluripotency marker. For AP staining, plates containing ESCs cultured in the presence or absence of LIF were washed with PBS with $\mathrm{Ca}^{+2}$ and $\mathrm{Mg}^{+2}$ (Dulbecco's Phosphate Buffered Saline, D8662; Sigma-Aldrich), and the staining was performed according to the instructions provided by the manufacturer (86R-1KT; Sigma-A ldrich). The plates were air-dried and were kept at RT.

\section{Colony formation assay}

To analyse the capability to form colonies from single cells of knockdown and KO lines, colony formation assays were performed as described previously (Fidalgo et al, 2016) with some modifications. Briefly, 800 cells were seeded in six-well plates coated with $0.1 \%$ gelatin and cultured in ESCS standard medium. $24 \mathrm{~h}$ later, the medium was changed to normal ESC medium or ESC medium without LIF. The media were changed every other day onwards. After $4 \mathrm{~d}$, AP staining was performed as described above. The number of colonies was counted and classified according to their pluripotent state: undifferentiated (AP positive), partially differentiated (partially AP positive), or differentiated (AP negative).

\section{Western blot analysis}

Whole cell extracts were obtained by lysing ESCs subjected to the different treatments with RIPA Lysis Buffer System (sc-24948; Santa Cruz Biotechnology) with freshly added protease and phosphatase 
inhibitors (sc-24948; Santa Cruz Biotechnology). Lysates were incubated during $30 \mathrm{~min}$ at $4^{\circ} \mathrm{C}$ and centrifuged for $10 \mathrm{~min}$ at $10,000 \mathrm{~g}$ at $4^{\circ} \mathrm{C}$ to remove cellular debris. Samples were kept at $-80^{\circ} \mathrm{C}$ until use.

Protein samples for Western blot analysis were prepared by adding Laemmli buffer and denaturing for $5 \mathrm{~min}$ at $95^{\circ} \mathrm{C}$ and loaded for resolution in a Novex WedgeWell 4-20\% tris-Glycine Gel (XP04205BOX; Invitrogen). For Western blotting, the following antibodies and dilutions were used: $\alpha$ BMAL1 (1:2,000, ab93806; Abcam), aPOU5F1 (1:4,000, sc-5279; Santa Cruz Biotechnology), aNANOG (1:2,000, A300-397A-2; Bethyl Laboratories), aZFP42 (1:1,000, sc-514643; Santa Cruz Biotechnology), aEZH2 (1:5,000, \#5246S; Cell Signaling Technology), aSUZ12 (1:1,000, sc-271325; Santa Cruz Biotechnology), and $\alpha$-ACTIN (1:2,000, sc-47778; Santa Cruz Biotechnology) or $\beta$-TUB ULIN (1:2,500, sc-55529; Santa Cruz Biotechnology) as loading controls.

\section{Immunofluorescence}

12,000 cells were seeded in wells of 48 -well plates coated with $0.1 \%$ gelatin and cultured in ESCs standard medium. After $72 \mathrm{~h}$, cells were fixed with $4 \%$ paraformaldehyde for $15 \mathrm{~min}$ in darkness at RT and washed two times with PBS (Dulbecco's Phosphate Buffered Saline, D8537; Sigma-Aldrich). Then, cells were permeabilizated with $0.25 \%$ Triton X-100 (T8787; Sigma-Aldrich) diluted in PBS for 5 min at RT followed by two times of PBS washes and blocking with 10\% BSA (BP9702-100; Thermo Fisher Scientific) diluted in PBS for 30 min at $37^{\circ} \mathrm{C}$. The antibodies $\alpha$ POU5F1 (1:1,500, sc-5279; Santa Cruz Biotechnology), aSSEA1-488 (1:1,000, MA1-022-D488; Thermo Fisher Scientific), aZFP281 (1:1,000, sc-166933; Santa Cruz Biotechnology), $\alpha$ NANOG (1:500, sc-374103; Santa Cruz Biotechnology), and $\alpha$ SOX2 (1:1,000, sc-365823; Santa Cruz Biotechnology) were diluted in 3\% BSA and incubated overnight in darkness at $4^{\circ} \mathrm{C}$. To detect nonlabelled primary antibodies, a rhodamine red-x secondary antibody was used (715-295-151; Jackson ImmunoResearch) for $1 \mathrm{~h}$ in darkness at RT. Nuclei were stained with DAPI (4',6-diamidino-2-phenilindole, D9542; Sigma-Aldrich).

\section{Microscopy}

Immunofluorescence images were taken with a Leica DMI 6000 inverted microscope with a $40 \times$ or $20 \times$ objective. The image processing was performed using the Leica Application Suite $X$, v3.0.11.20652. Bright-field and colour images were taken with a camera Olympus DP72 coupled to an inverted Olympus IX51 microscope.

\section{Genomic edition using CRISPR/Cas9 technology}

Bmal1 KO ESCs were generated with CRISPR/Cas9 editing tool as previously described (Ran et al, 2013). Briefly, an sgRNA targeting the exon 5 of the Bmal1 gene was designed using the webtool available at http://crispr.mit.edu and was cloned into the pSpCas9(BB)-2APuro (PX459) V2.0 vector. After verifying the correct sequence by SA NGER sequencing, ESCs were transfected with the sgRNA-containing plasmid using the jetPRIME kit (114-07; Polyplus) following the manufacturer's instructions. Transfected cells were seeded at clonal density and selected with $2 \mu \mathrm{g} / \mathrm{ml}$ puromycin to obtain single colonies suitable for manual picking. Several clones were isolated, and the identity of a KO cell line was confirmed by Sanger sequencing and Western blot analysis. pSpCas9(BB)-2A-Puro (PX459) V2.0 was a gift from Feng Zhang (plasmid \#62988; Addgene; http:/ / n2t.net/addgene:62988; RRID: Addgene 62988) (Ran et al, 2013).

\section{DNA extraction and genotyping}

Cells were resuspended in Cell Lysis Solution (158908; QIAGEN) and treated with RNAse A (EN0531; Thermo Fisher Scientific) for $1 \mathrm{~h}$. Then, Protein Precipitation Solution (158912; QIAGEN) was added and samples were centrifuged at $16,000 \mathrm{~g}$ for $10 \mathrm{~min}$. Once the samples were clean of protein, the DNA was precipitated using isopropanol and washed with ethanol 70\%. Finally, genomic DNA was resuspended in DNA Hydration Solution (158916; QIAGEN) overnight at RT.

A three-primer strategy was designed to detect homozygotic deletions in the CRISPR/Cas9-transfected clones as schematized in Fig S2A. Oligo sequences are shown in Table S2.

\section{RA differentiation assay}

For this assay, NG4 ESCs containing a GFP reporter under the control of the pluripotency-related Nanog promoter were used. 50,000 cells transfected with shRNA against Bmal1 or Luciferase as a control were seeded in 12-well plates coated with $0.1 \%$ gelatine and cultured at $37^{\circ} \mathrm{C}$ with $5 \% \mathrm{CO}_{2}$ in ESCs standard medium. After $24 \mathrm{~h}$, the medium was maintained as control or switched for standard medium without LIF containing $10 \mathrm{nM}$ of RA. After $48 \mathrm{~h}$ in RAcontaining medium, the cells were analysed by flow cytometry in a BD Accuri C6 Plus instrument (BD Biosciences).

\section{EB differentiation assay}

EBs were formed by aggregation of ESCS in suspension in a lowattachment petri dish as previously described (Doetschman et al, 1985). Briefly, ESCs transfected with shRNA against Bmal1 and Luciferase as control were seeded at 155,000 cells/ml in lowattachment plates and cultured at $37^{\circ} \mathrm{C}$ with $5 \% \mathrm{CO}_{2}$ in standard ESC medium without LIF. The medium was refreshed every other day and samples were collected at day 6 of EB formation.

\section{Gastruloid aggregation assay}

Gastruloid aggregation assays were performed as previously described (Baillie-Johnson et al, 2015; Beccari et al, 2018). Briefly, for the generation of aggregates, an average of 400 ESCs were resuspended in $40 \mu \mathrm{l}$ of N2B27 medium (50\% DMEM/F12 [Nutrient Mixture F-12 Ham, D6421; Sigma-Aldrich], 50\% Neurobasal Medium [21103-049; Gibco] containing 1× B27 and N2 supplements [17504-044 and 17502-048, respectively; Gibco], 2 mM L-glutamine [SH30034.01; GE Healthcare], 1× penicillin/streptomycin [15140; Gibco]), seeded in non-tissue-culture treated u-bottomed 96-well plates and cultured at $37^{\circ} \mathrm{C}$ with $5 \% \mathrm{CO}_{2} .48 \mathrm{~h}$ after seeding, a $3-\mu \mathrm{M}$ CHIR stimuli was applied for $24 \mathrm{~h}$, with media being changed every day. At $120 \mathrm{~h}$, pictures were taken and 16 gastruloids were pooled in $200 \mu \mathrm{l}$ of Trizol for RNA extraction as detailed above. 
To calculate Bmal1 WT and Bmal1 KO gastruloid generation efficiency, the percentage of positive gastruloid-like structures in each experiment was compared ( $n=14$ groups of eight aggregates each). Gastruloid size measurement was performed using ImageJ Software, quantifying 112 gastruloids per condition.

\section{Mesendoderm differentiation assay}

Mesendoderm differentiation assay was performed as previously described (Thomson et al, 2011). Briefly, Bmal1 KO or WT ESCs were maintained for a couple of passages in N2B27 supplemented with LIF, $10 \mathrm{ng} / \mathrm{ml} \mathrm{BMP4}$ (11330852; Gibco), and $1 \mu \mathrm{M}$ MEK inhibitor PD 0325901 (PZ0162; Sigma-Aldrich). Then, $1.5 \times 10^{4}$ cells $/ \mathrm{cm}^{2}$ were seeded on gelatin-coated plates in N2B27 medium. $48 \mathrm{~h}$ later, the medium was switched to N2B27 supplemented with CHIR99021 $3 \mu \mathrm{M}$ (SML1046; Sigma-Aldrich) to induce mesendoderm differentiation. To diminish OXPHOS activity, the cells were treated with rotenone and antimycin $\mathrm{A}$ at a concentration of $50 \mathrm{nmol} / \mathrm{l}$ each. Rotenone is an inhibitor of the electron transport chain (ETC) complex I, and antimycin A is a complex III inhibitor. After $36 \mathrm{~h}$, total RNA was extracted using the E.Z.N.A. Total RNA Kit I kit as described above.

\section{Teratoma formation}

For teratoma formation, all animal procedures were performed in accordance with the University of Extremadura's Institutional Animal Care and Use Committee. Eight female Swiss nude mice (Charles River Laboratory) at 5 wk of age were used for this assay. Bmal1 WT and Bmal1 KO ESCs were grown and passaged at $80 \%$ confluence. At the time of injection, the cells were washed with PB S-EDTA, trypsinized, and counted. Aliquots of $200 \mu \mathrm{l}$ of 1:4 Matrigel/ PBS containing $1 \times 10^{6}$ Bmal1 WT or Bmal1 $\mathrm{KO}$ cells were injected subcutaneously in each mouse flank. Teratomas were excised 3-4 wk after injection, measured, and processed for teratoma biopsy histology. Tumours were fixed overnight in formalin, embedded in paraffin, sectioned at $5 \mu \mathrm{m}$, and stained with haematoxylin and eosin. Histological evaluation was performed using a Nikon TE2000U microscope and ACT-1 Software.

\section{RNA-seq generation and analysis}

RNA extraction method for RNA-seq samples is described above. Reads from the sequencing of biological replicates of WT and Bmal1 KO ESCS were aligned to the mouse genome (GRCm38, mm10) using TopHat (v2.1.1) and Bowtie2 (v2.3.0) with the default parameters settings, providing a GTF containing known transcripts $(-G)$. Transcript assembly and differential expression analysis were performed by Cufflinks (v2.2.1), expression of transcripts sharing each gene_id was quantified as FPKM (fragments per million mapped reads), and significance of differential expression tests was determined with the Benjamini-Hochberg correction for multiple testing. Differentially expressed genes were identified as genes having more than twofold change in expression in Bmal1 KO compared with WT cells and an FDR value of less than 0.05 .

\section{GO, KEGG pathway and Network2Canvas analyses}

GO and KEGG analyses were performed with DAVID functional annotation tool at http:/ / david.abcc.ncifcrf.gov/tools.jsp (Huang da et al, 2009) with a reference list including all Mus musculus genes from NCBI. Network2Canvas (Network Visualization on a Canvas with Enrichment Analysis) (http:/ / www.maayanlab.net/ N2C/) (Tan et al, 2013) was used to interrogate the correlation of gene expression changes in Bmal1 KO ESCs with transcriptional changes upon lossof-function of transcription regulators from published datasets.

\section{GSEA}

GSEA (v3.0, accessible at http://software.broadinstitute.org/gsea/ index.jsp) was used to analyse the enrichment differences between Bmal1 WT and KO cells. Briefly, the analysis was performed running with 1,000 permutations and gene_set permutation type. $t$ test metric to rank genes and weighted_p2 for enrichment statistics were used. Enrichment plot, normalized enrichment score, statistical significance ( $P$-value), and FDR were calculated by the software.

\section{Extracellular metabolic flux analysis}

45,000 Bmal1 WT and KO ESCs were seeded in Seahorse XFp Cell Culture Miniplates (103025-100; Agilent) coated with $0.1 \%$ gelatin and cultured at $37^{\circ} \mathrm{C}$ with $5 \% \mathrm{CO}_{2}$ in standard ESCs medium for $24 \mathrm{~h}$. OCR and extracellular acidification rate were measured using Seahorse XFp Cell Mito Stress Test Kit (103010-100; Agilent) and Seahorse XFp Glycolitic Rate Assay Kit (103346-100; Agilent), respectively, following the manufacturer's instructions. Briefly, the cells were incubated 45-60 min in Seahorse XF DMEM Medium, pH 7.4 (103575-100; Agilent), supplemented with pyruvate $1 \mathrm{mM}$, L-glutamine $2 \mathrm{mM}$, glucose $10 \mathrm{mM}$, and Hepes $5 \mathrm{mM}$ (this only for the Glycolytic Rate Assay), and mitochondrial function was analysed in Seahorse XFp Analyser (Agilent).

Proton leak and coupling efficiency parameters were calculated using the OCRs data obtained with the Seahorse XFp Cell Mito Stress Test Kit (103010-100; Agilent). The assay involves the use of three different drugs: oligomycin, which decreases the electron flow through the ETC, resulting in a mitochondrial respiration reduction; carbonyl cyanide-4 phenylhydrazone (FCCP), which restores the electron flow through the ETC obtaining the maximal oxygen consumption; and AA/Rotenone, which completely blocks mitochondrial respiration and allows the calculation of nonmitochondrial oxygen consumption. Proton leak was calculated as the minimum OCR measurement after oligomycin injection minus the non-mitochondrial oxygen consumption, determined as the median of the OCR data after AA/Rotenone addition. Coupling efficiency was calculated as the ATP-linked OCR divided by the basal mitochondrial OCR and multiplied by 100 .

\section{mtROS analysis}

The same number of Bmal1 WT and KO ESCS was seeded in 12-well plates in ESC standard medium. $24 \mathrm{~h}$ later, the cells were stained with MitoSOX Red mitochondrial superoxide indicator for live-cell 
imaging (M36008; Invitrogen) following the manufacturer's instructions. Briefly, the samples used for cytometry analysis were dissociated with trypsin (SH30236.01; GE Healthcare) and washed with PBS with $\mathrm{Ca}^{+2}$ and $\mathrm{Mg}^{+2}$ (Dulbecco's Phosphate Buffered Saline, D8662; Sigma-Aldrich). Then, the cells were incubated for $10 \mathrm{~min}$ in $5 \mu \mathrm{M}$ MitosOX reagent working solution in PBS or only in PBS as a control and analysed by flow cytometry in a BD Accuri C6 Plus instrument (BD Biosciences).

Cells used for imaging were treated with $5 \mu \mathrm{M}$ MitoSOX reagent working solution in PBS on plates for $10 \mathrm{~min}$ at $37^{\circ} \mathrm{C}$. Then, nuclei were stained with DAPI, and images were acquired with a Leica DMI 6000 inverted microscope with a $20 \times$ objective.

\section{Statistical analysis}

Differences with two-sided $P<0.05$ were deemed statistically significant, as evaluated using the $t$ test. Results are reported as mean \pm SD or SEM as indicated in the figure legends.

\section{Data Availability}

RNA-seq datasets generated in this study are deposited at Gene Expression Omnibus (accession number GSE133908).

\section{Supplementary Information}

Supplementary Information is available at https://doi.org/10.26508/lsa. 201900534.

\section{Acknowledgements}

This research was funded by Spanish Agencia Estatal de Investigación and co-funded by the FEDER Program of the European Union (BFU2016-80899-P to M Fidalgo and RTI2018-096708-J-I00 to D Guallar) (AEI/FEDER, EU), the Xunta de Galicia-Consellería de Cultura, Educación e Ordenación Universitaria (ED431F 2016/016 to M Fidalgo), and the Fundación Ramón Areces (2016-PO025 to M Fidalgo). This work was also supported by BFU2017-85547-P grant from the Ministry of Economy, IB18014 and GR15164 from Junta de Extremadura to JM Carvajal-Gonzalez. M Fidalgo and JM Carvajal-Gonzalez are recipients of Ramón y Cajal awards (RYC-2014-16779 and RYC-2015-17867, respectively) from the Ministry of Economy and Competitiveness (MINECO) of Spain. A Fuentes-Iglesias, V Garcia-Outeiral, and A Escudero are recipients of fellowships (MINECO, BES-2017-082007 to A Fuentes-Iglesias and Ministerio de Ciencia, Innovación y Universidades; FPU17/01131 to V GarciaOuteiral and FPU2018/01246 to A Escudero).

\section{Author Contributions}

C Ameneiro: conceptualization, resources, formal analysis, validation, investigation, visualization, methodology, and writing-original draft.

T Moreira: resources, formal analysis, validation, investigation, and methodology.

A Fuentes-Iglesias: resources, data curation, software, formal analysis, validation, investigation, and visualization.

A Coego: formal analysis, validation, and investigation.
V Garcia-Outeiral: formal analysis, validation, investigation, visualization, and methodology.

A Escudero: formal analysis, validation, investigation, visualization, and methodology.

D Torrecilla: formal analysis, investigation, visualization, and methodology.

S Mulero-Navarro: resources, formal analysis, validation, investigation, visualization, and methodology.

JM Carvajal-Gonzalez: resources, formal analysis, funding acquisition, validation, investigation, visualization, and methodology.

D Guallar: conceptualization, resources, data curation, formal analysis, supervision, funding acquisition, validation, investigation, visualization, methodology, project administration, and writing-original draft, review, and editing.

M Fidalgo: conceptualization, resources, data curation, formal analysis, supervision, funding acquisition, validation, investigation, visualization, methodology, project administration, and writing-original draft, review, and editing.

\section{Conflict of Interest Statement}

The authors declare that they have no conflict of interest.

\section{References}

Alvarez JD, Hansen A, Ord T, Bebas P, Chappell PE, Giebultowicz JM, Williams C, Moss S, Sehgal A (2008) The circadian clock protein BMAL1 is necessary for fertility and proper testosterone production in mice. J Biol Rhythms 23: 26-36. doi:10.1177/0748730407311254

Ang YS, Tsai SY, Lee DF, Monk J, Su J, Ratnakumar K, Ding J, Ge Y, Darr H, Chang B, et al (2011) Wdr5 mediates self-renewal and reprogramming via the embryonic stem cell core transcriptional network. Cell 145: 183-197. doi:10.1016/j.cell.2011.03.003

Baillie-Johnson P, van den Brink SC, Balayo T, Turner DA, Martinez Arias A (2015) Generation of aggregates of mouse embryonic stem cells that show symmetry breaking, polarization and emergent collective behaviour in vitro. J Vis Exp. doi:10.3791/53252

Beccari L, Moris N, Girgin M, Turner DA, Baillie-Johnson P, Cossy AC, Lutolf MP, Duboule D, Arias AM (2018) Multi-axial self-organization properties of mouse embryonic stem cells into gastruloids. Nature 562: 272-276. doi:10.1038/s41586-018-0578-0

Bechtold DA, Loudon AS (2013) Hypothalamic clocks and rhythms in feeding behaviour. Trends Neurosci 36: 74-82. doi:10.1016/j.tins.2012.12.007

Ben-Porath I, Thomson MW, Carey VJ, Ge R, Bell GW, Regev A, Weinberg RA (2008) An embryonic stem cell-like gene expression signature in poorly differentiated aggressive human tumors. Nat Genet 40 : 499-507. doi:10.1038/ng.127

Boden MJ, Varcoe TJ, Voultsios A, Kennaway DJ (2010) Reproductive biology of female Bmal1 null mice. Reproduction 139: 1077-1090. doi:10.1530/rep09-0523

Brookes E, de Santiago I, Hebenstreit D, Morris KJ, Carroll T, Xie SQ, Stock JK Heidemann M, Eick D, Nozaki N, et al (2012) Polycomb associates genome-wide with a specific RNA polymerase II variant, and regulates metabolic genes in ESCs. Cell Stem Cell 10: 157-170. doi:10.1016/ j.stem.2011.12.017

Bunger MK, Wilsbacher LD, Moran SM, Clendenin C, Radcliffe LA, Hogenesch JB, Simon MC, Takahashi JS, Bradfield CA (2000) Mop3 is an essential component of the master circadian pacemaker in mammals. Cell 103: 1009-1017. doi:10.1016/s0092-8674(00)00205-1 
Cadenas S (2018) Mitochondrial uncoupling, ROS generation and cardioprotection. Biochim Biophys Acta Bioenerg 1859: 940-950. doi:10.1016/j.bbabio.2018.05.019

Chen $\mathrm{X}, \mathrm{Xu} \mathrm{H}$, Yuan $\mathrm{P}$, Fang F, Huss M, Vega VB, Wong E, Orlov YL, Zhang W, Jiang J, et al (2008) Integration of external signaling pathways with the core transcriptional network in embryonic stem cells. Cell 133: 1106-1117. doi:10.1016/j.cell.2008.04.043

Cliff TS, Dalton S (2017) Metabolic switching and cell fate decisions: Implications for pluripotency, reprogramming and development. Curr Opin Genet Dev 46: 44-49. doi:10.1016/j.gde.2017.06.008

Cong L, Ran FA, Cox D, Lin S, Barretto R, Habib N, Hsu PD, Wu X, Jiang W, Marraffini LA, et al (2013) Multiplex genome engineering using CRISPR/Cas systems. Science 339: 819-823. doi:10.1126/science.1231143

Dahan P, Lu V, Nguyen RMT, Kennedy SAL, Teitell MA (2019) Metabolism in pluripotency: Both driver and passenger? J Biol Chem 294: 5420-5429. doi:10.1074/jbc.tm117.000832

Di Croce L, Helin K (2013) Transcriptional regulation by Polycomb group proteins. Nat Struct Mol Biol 20: 1147-1155. doi:10.1038/nsmb.2669

Dierickx P, Van Laake LW, Geijsen N (2018) Circadian clocks: From stem cells to tissue homeostasis and regeneration. EMBO Rep 19: 18-28. doi:10.15252/embr.201745130

Dierickx P, Vermunt MW, Muraro MJ, Creyghton MP, Doevendans PA, van Oudenaarden A, Geijsen N, Van Laake LW (2017) Circadian networks in human embryonic stem cell-derived cardiomyocytes. EMBO Rep 18: 1199-1212. doi:10.15252/embr.201743897

Doetschman TC, Eistetter H, Katz M, Schmidt W, Kemler R (1985) The in vitro development of blastocyst-derived embryonic stem cell lines: Formation of visceral yolk sac, blood islands and myocardium. J Embryol Exp Morphol 87: 27-45.

Fidalgo M, Huang X, Guallar D, Sanchez-Priego C, Valdes VJ, Saunders A, Ding J, Wu WS, Clavel C, Wang I (2016) Zfp281 coordinates opposing functions of Tet1 and Tet2 in pluripotent states. Cell Stem Cell 19: 355-369. doi:10.1016/j.stem.2016.05.025

Galan-Caridad JM, Harel S, Arenzana TL, Hou ZE, Doetsch FK, Mirny LA, Reizis B (2007) Zfx controls the self-renewal of embryonic and hematopoietic stem cells. Cell 129: 345-357. doi:10.1016/j.cell.2007.03.014

Gallardo A, Molina A, Asenjo HG, Martorell-Marugán J, Montes R, Ramos-Mejia V, Sanchez-Pozo A, Carmona-Sáez P, Landeira D (2020) The molecular clock protein Bmal1 regulates cell differentiation in mouse embryonic stem cells. Life Sci Alliance 3. doi:10.26508/Isa.201900535

Gingold JA, Fidalgo M, Guallar D, Lau Z, Sun Z, Zhou H, Faiola F, Huang X, Lee DF, Waghray A, et al (2014) A genome-wide RNAi screen identifies opposing functions of Snai1 and Snai2 on the Nanog dependency in reprogramming. Mol Cell 56: 140-152. doi:10.1016/j.molcel.2014.08.014

Hatanaka F, Matsubara C, Myung J, Yoritaka T, Kamimura N, Tsutsumi S, Kanai A, Suzuki Y, Sassone-Corsi P, Aburatani H, et al (2010) Genome-wide profiling of the core clock protein BMAL1 targets reveals a strict relationship with metabolism. Mol Cell Biol 30: 5636-5648. doi:10.1128/ mcb.00781-10

Huang DW, Sherman BT, Lempicki RA (2009) Bioinformatics enrichment tools: Paths toward the comprehensive functional analysis of large gene lists. Nucleic Acids Res 37: 1-13. doi:10.1093/nar/gkn923

Jinek M, Chylinski K, Fonfara I, Hauer M, Doudna JA, Charpentier E (2012) A programmable dual-RNA-guided DNA endonuclease in adaptive bacterial immunity. Science 337: 816-821. doi:10.1126/ science. 1225829

Kim H, Jang H, Kim TW, Kang BH, Lee SE, Jeon YK, Chung DH, Choi J, Shin J, Cho EJ, et al (2015) Core pluripotency factors directly regulate metabolism in embryonic stem cell to maintain pluripotency. Stem Cells 33: 2699-2711. doi:10.1002/stem.2073

Kondratov RV, Kondratova AA, Gorbacheva VY, Vykhovanets OV, Antoch MP (2006) Early aging and age-related pathologies in mice deficient in
BMAL1, the core componentof the circadian clock. Genes Dev 20: 1868-1873. doi:10.1101/gad.1432206

Kowalska E, Moriggi E, Bauer C, Dibner C, Brown SA (2010) The circadian clock starts ticking at a developmentally early stage. J Biol Rhythms 25: 442-449. doi:10.1177/0748730410385281

Lee DF, Su J, Ang YS, Carvajal-Vergara X, Mulero-Navarro S, Pereira CF, Gingold J, Wang HL, Zhao R, Sevilla A, et al (2012) Regulation of embryonic and induced pluripotency by aurora kinase-p53 signaling. Cell Stem Cell 11: 179-194. doi:10.1016/j.stem.2012.05.020

Liu Y, Johnson BP, Shen AL, Wallisser JA, Krentz KJ, Moran SM, Sullivan R, Glover E, Parlow AF, Drinkwater NR, et al (2014) Loss of BMAL1 in ovarian steroidogenic cells results in implantation failure in female mice. Proc Natl Acad Sci U S A 111: 14295-14300. doi:10.1073/ pnas.1209249111

Lopez-Minguez J, Gomez-Abellan P, Garaulet M (2016) Circadian rhythms, food timing and obesity. Proc Nutr Soc 75: 501-511. doi:10.1017/ s0029665116000628

Lu C, Yang Y, Zhao R, Hua B, Xu C, Yan Z, Sun N, Qian R (2016) Role of circadian gene Clock during differentiation of mouse pluripotent stem cells. Protein Cell 7: 820-832. doi:10.1007/s13238-0160319-9

Matsuzuka T, Ozawa M, Nakamura A, Ushitani A, Hirabayashi M, Kanai Y (2005) Effects of heat stress on the redox status in the oviduct and early embryonic development in mice. J Reprod Dev 51:281-287. doi:10.1262/ jrd.16089

McAlpine CS, Swirski FK (2016) Circadian influence on metabolism and inflammation in atherosclerosis. Circ Res 119: 131-141. doi:10.1161/ circresaha.116.308034

Nanayakkara GK, Wang H, Yang X (2019) Proton leak regulates mitochondrial reactive oxygen species generation in endothelial cell activation and inflammation: A novel concept. Arch Biochem Biophys 662: 68-74. doi:10.1016/j.abb.2018.12.002

Paulose JK, Rucker EB 3rd, Cassone VM (2012) Toward the beginning of time: Circadian rhythms in metabolism precede rhythms in clock gene expression in mouse embryonic stem cells. PLoS One 7: e49555. doi:10.1371/journal.pone.0049555

Ran FA, Hsu PD, Wright J, Agarwala V, Scott DA, Zhang F (2013) Genome engineering using the CRISPR-Cas9 system. Nat Protoc 8: 2281-2308. doi:10.1038/nprot.2013.143

Ratajczak CK, Asada M, Allen GC, McMahon DG, Muglia LM, Smith D, Bhattacharyya S, Muglia LJ (2012) Generation of myometrium-specific Bmal1 knockout mice for parturition analysis. Reprod Fertil Dev 24: 759-767. doi:10.1071/rd11164

Reinke H, Asher G (2019) Crosstalk between metabolism and circadian clocks. Nat Rev Mol Cell Biol 20: 227-241. doi:10.1038/s41580-018-0096-9

Rudic RD, McNamara P, Curtis AM, Boston RC, Panda S, Hogenesch JB, Fitzgerald GA (2004) BMAL1 and CLOCK, two essential components of the circadian clock, are involved in glucose homeostasis. PLOS Biol 2: e377. doi:10.1371/journal.pbio.0020377

Saxena MT, Aton SJ, Hildebolt C, Prior JL, Abraham U, Piwnica-Worms D, Herzog ED (2007) Bioluminescence imaging of period1 gene expression in utero. Mol Imaging 6: 68-72. doi:10.2310/ 7290.2007 .00003

Schaniel C, Ang YS, Ratnakumar K, Cormier C, James T, Bernstein E, Lemischka IR, Paddison PJ (2009) Smarcc1/Baf155 couples self-renewal gene repression with changes in chromatin structure in mouse embryonic stem cells. Stem Cells 27: 2979-2991. doi:10.1002/stem.223

Stefkova K, Prochazkova J, Pachernik J (2015) Alkaline phosphatase in stem cells. Stem Cells Int 2015: 628368. doi:10.1155/2015/628368

Stewart SA, Dykxhoorn DM, Palliser D, Mizuno H, Yu EY, An DS, Sabatini DM, Chen IS, Hahn WC, Sharp PA, et al (2003) Lentivirus-delivered stable 
gene silencing by RNAi in primary cells. RNA 9: 493-501. doi:10.1261/ rna.2192803

Takahashi JS (2017) Transcriptional architecture of the mammalian circadian clock. Nat Rev Genet 18: 164-179. doi:10.1038/nrg.2016.150

Tamura H, Takasaki A, Miwa I, Taniguchi K, Maekawa R, Asada H, Taketani T, Matsuoka A, Yamagata Y, Shimamura K, et al (2008) Oxidative stress impairs oocyte quality and melatonin protects oocytes from free radical damage and improves fertilization rate. I Pineal Res 44: 280-287. doi:10.1111/j.1600-079x.2007.00524.x

Tan CM, Chen EY, Dannenfelser R, Clark NR, Ma'ayan A (2013) Network2Canvas: Network visualization on a canvas with enrichment analysis. Bioinformatics 29: 1872-1878. doi:10.1093/bioinformatics/btt319

Teslaa T, Teitell MA (2015) Pluripotent stem cell energy metabolism: An update. EMBO / 34: 138-153. doi:10.15252/embj.201490446

Thomson M, Liu SJ, Zou LN, Smith Z, Meissner A, Ramanathan S (2011) Pluripotency factors in embryonic stem cells regulate differentiation into germ layers. Cell 145: 875-889. doi:10.1016/j.cell.2011.05.017

Umemura Y, Koike N, Matsumoto T, Yoo SH, Chen Z, Yasuhara N, Takahashi JS, Yagita K (2014) Transcriptional program of Kpna2/Importin-alpha2 regulates cellular differentiation-coupled circadian clock development in mammalian cells. Proc Natl Acad Sci U S A 111: E5039-E5048. doi:10.1073/pnas.1419272111

Umemura Y, Koike N, Ohashi M, Tsuchiya Y, Meng QJ, Minami Y, Hara M, Hisatomi M, Yagita K (2017) Involvement of posttranscriptional regulation of clock in the emergence of circadian clock oscillation during mouse development. Proc Natl Acad Sci U S A 114: E7479-E7488. doi:10.1073/pnas.1703170114

Weger M, Diotel N, Dorsemans AC, Dickmeis T, Weger BD (2017) Stem cells and the circadian clock. Dev Biol 431: 111-123. doi:10.1016/ j.ydbio.2017.09.012

Wu J, Ocampo A, Belmonte JCI (2016) Cellular metabolism and induced pluripotency. Cell 166: 1371-1385. doi:10.1016/j.cell.2016.08.008
Wu Y, Tang D, Liu N, Xiong W, Huang H, Li Y, Ma Z, Zhao H, Chen P, Qi X, et al (2017) Reciprocal regulation between the circadian clock and hypoxia signaling at the genome level in mammals. Cell Metab 25: 73-85. doi:10.1016/j.cmet.2016.09.009

Xu J, Li Y, Wang Y, Xu Y, Zhou C (2016) Loss of Bmal1 decreases oocyte fertilization, early embryo development and implantation potential in female mice. Zygote 24: 760-767. doi:10.1017/s0967199416000083

Xu J, Wang Y, Xu Y, Zhou C (2017) Clock gene Bmal1 in mice embryo is dispensable for early embryo development but critical for live birth. Biol Rhythm Res 48: 831-836. doi:10.1080/09291016.2017.1307975

Yagita K, Horie K, Koinuma S, Nakamura W, Yamanaka I, Urasaki A, Shigeyoshi Y, Kawakami K, Shimada S, Takeda J, et al (2010) Development of the circadian oscillator during differentiation of mouse embryonic stem cells in vitro. Proc Natl Acad Sci U S A 107: 3846-3851. doi:10.1073/ pnas.0913256107

Yagita K, Tamanini F, van Der Horst GT, Okamura H (2001) Molecular mechanisms of the biological clock in cultured fibroblasts. Science 292: 278-281. doi:10.1126/science.1059542

Yang G, Chen L, Grant GR, Paschos G, Song WL, Musiek ES, Lee V, McLoughlin SC, Grosser T, Cotsarelis G, et al (2016) Timing of expression of the core clock gene Bmal1 influences its effects on aging and survival. Sci Transl Med 8: 324ra316. doi:10.1126/scitranslmed.aad3305

Zhang J, Zhao J, Dahan P, Lu V, Zhang C, Li H, Teitell MA (2018) Metabolism in pluripotent stem cells and early mammalian development. Cell Metab 27: 332-338. doi:10.1016/j.cmet.2018.01.008

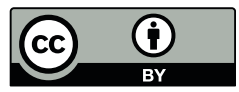

License: This article is available under a Creative Commons License (Attribution 4.0 International, as described at https://creativecommons.org/ licenses/by/4.0/). 